\title{
LONG RANGE CORRELATION INEQUALITIES FOR MASSLESS EUCLIDEAN FIELDS
}

\author{
JOSEPH G. CONLON AND ARASH FAHIM
}

\begin{abstract}
In this paper, new correlation inequalities are obtained for massless Euclidean fields on the $d$ dimensional integer lattice. Some of the inequalities have been obtained previously, in the case where the Lagrangian is a very small perturbation of a quadratic, using the renormalization group method. The results of the present paper apply provided the Lagrangian is uniformly convex. They therefore hold for the Coulomb dipole gas in which particle density can be of order 1 . The approach of the present paper is based on the methodology of Naddaf-Spencer, which relates second moment correlation functions for the Euclidean field to expectations of Green's functions for parabolic PDE with random coefficients.
\end{abstract}

\section{Introduction}

In this paper, we shall prove some new correlation inequalities for certain translation invariant probability measures on fields $\phi: \mathbf{Z}^{d} \rightarrow \mathbf{R}$, where $\mathbf{Z}^{d}$ is the $d$ dimensional integer lattice. These measures are determined by a $C^{2}$ uniformly convex function $V: \mathbf{R}^{d} \rightarrow \mathbf{R}$. They can be formally written as the limit $m \rightarrow 0$ of the weighted Euclidean measure on $\mathbf{R}^{\infty}$ given by

$$
\exp \left[-\sum_{x \in \mathbf{Z}^{d}} V(\nabla \phi(x))+m^{2} \phi(x)^{2} / 2\right] \prod_{x \in \mathbf{Z}^{d}} d \phi(x) / \text { normalization. }
$$

In (1.1), we take $\nabla$ to be the discrete gradient operator defined by

$$
\nabla \phi(x)=\left(\nabla_{1} \phi(x), \ldots, \nabla_{d} \phi(x)\right), \quad \nabla_{i} \phi(x)=\phi\left(x+\mathbf{e}_{i}\right)-\phi(x),
$$

where the vector $\mathbf{e}_{i} \in \mathbf{Z}^{d}$ has 1 as the $i$ th coordinate and 0 for the other coordinates, $1 \leq i \leq d$. Here we shall always consider $\nabla$ to be a $d$ dimensional

Received April 7, 2015; received in final form August 26, 2015.

2010 Mathematics Subject Classification. 35R60, 82B20, 82B28. 
column operator, with adjoint $\nabla^{*}$ which is a $d$ dimensional row operator. The existence of the massive measures (1.1) for $m>0$, and limiting massless measures as $m \rightarrow 0$, was established by Funaki and Spohn [20] for functions $V(\cdot)$ which satisfy a quadratic form inequality $\lambda I_{d} \leq V^{\prime \prime}(\cdot) \leq \Lambda I_{d}$, where $\lambda$, $\Lambda$ are positive constants. The massless measure is a probability measure on gradient fields $\omega: \mathbf{Z}^{d} \rightarrow \mathbf{R}^{d}$, where formally $\omega(x)=\nabla \phi(x), x \in \mathbf{Z}^{d}$. In the case $d=1$, the variables $\omega(x), x \in \mathbf{Z}$, are independent identically distributed. If $d \geq 3$, the gradient field measure induces a measure on fields $\phi: \mathbf{Z}^{d} \rightarrow \mathbf{R}$, which is simply the limit of the massive measures (1.1) as $m \rightarrow 0$. For $d=1,2$, the $m \rightarrow 0$ limit of the measures (1.1) on fields $\phi: \mathbf{Z}^{d} \rightarrow \mathbf{R}$ does not exist.

The main technical tool in the existence proof for the measures (1.1) is the Brascamp-Lieb inequality [4]. This inequality is a type of Poincaré inequality for probability measures on $\mathbf{R}^{n}$ with weight $\exp [-W(\phi)] d \phi$, where $\phi=\left[\phi_{1}, \ldots, \phi_{n}\right]$ and $W: \mathbf{R}^{n} \rightarrow \mathbf{R}$ is a $C^{2}$ convex function. The BrascampLieb inequality gives a bound on the variance of a $C^{1}$ function $g: \mathbf{R}^{n} \rightarrow \mathbf{R}$ in terms of an expectation of its gradient, $d g: \mathbf{R}^{n} \rightarrow \mathbf{R}^{n}$. Letting $\langle\cdot\rangle$ denote expectation and $\left[\cdot, \cdot \cdot\right.$ Euclidean inner product on $\mathbf{R}^{n}$, then the inequality is given by

$$
\operatorname{Var}[g] \leq\left\langle\left[d g, W^{\prime \prime}(\cdot)^{-1} d g\right]\right\rangle .
$$

One can apply the inequality (1.3) to the measure $(1.1)$. Letting $[\cdot, \cdot]$ now denote the Euclidean inner product on functions $f: \mathbf{Z}^{d} \rightarrow \mathbf{R}$ and $\Delta$ the lattice Laplacian, one obtains [36] the inequality

$$
\langle\exp \{[f, \phi]\}\rangle \leq \exp \left\{\frac{1}{2}\left[f,\left(-\lambda \Delta+m^{2}\right)^{-1} f\right]\right\} .
$$

It follows from (1.4) that for any $x \in \mathbf{Z}^{d}$ the expectation $\langle\phi(x) \phi(0)\rangle$ is finite for $m \geq 0$ if $d \geq 3$, and for $m>0$ if $d=2$. Even in the Gaussian case when $V(\cdot)$ is quadratic, the expectation $\langle\phi(x) \phi(0)\rangle$ does not exist if $m=0, d=2$. However, (1.4) implies that if $m=0, d=2$ then both $\langle\phi(x) \nabla \phi(0)\rangle$ and $\langle\nabla \phi(x) \nabla \phi(0)\rangle$ are finite.

The massless $m=0$ measures (1.1) occur in the study of Ginzburg-Landau $\nabla \phi$ interface models [20] and in the study of the lattice Coulomb dipole gas [5], [21]. In the case of the $d$ dimensional dipole gas, the function $V(\cdot)$ is given by

$$
V(z)=|z|^{2} / 2-a \sum_{j=1}^{d} \cos z_{j}, \quad z=\left[z_{1}, \ldots, z_{d}\right] \in \mathbf{R}^{d},
$$

where $a>0$ is the activity of the dipoles, corresponding to the density of dipoles on a given bond. If $\rho_{i}(x)$ is the charge density at $x \in \mathbf{Z}^{d}$ for dipoles on the bond $x \rightarrow x+\mathbf{e}_{i}, i=1, \ldots, d$, then one can see that the expectation $\left\langle\rho_{i}(x) \rho_{j}(y)\right\rangle_{\text {dipole }}$ of the Gibbs ensemble for the lattice Coulomb dipole gas is proportional to $\left\langle\sin \left[\nabla_{i} \phi(x)\right] \sin \left[\nabla_{j} \phi(y)\right]\right\rangle$ for the massless measure (1.1). 
A formal derivation of this is given in Appendix B. An important intuition concerning Coulomb gases is the idea of screening. That is that the interaction between widely separated charges decreases due to the presence of the other particles in the gas, at least at low densities. Thus, one expects that $\left|\left\langle\rho_{i}(x) \rho_{j}(y)\right\rangle_{\text {dipole }}\right|$ should be smaller than $\left|\left\langle\rho_{i}(x) \rho_{j}(y)\right\rangle_{x, y \text {,dipole }}\right|$

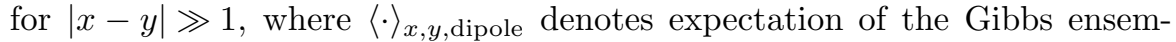
ble for just the two dipoles $x \rightarrow x+\mathbf{e}_{i}$ and $y \rightarrow y+\mathbf{e}_{j}$. One can easily see that $\left\langle\rho_{i}(x) \rho_{j}(y)\right\rangle_{x, y \text {,dipole }} \simeq\left(x_{i}-y_{i}\right)\left(x_{j}-y_{j}\right) /|x-y|^{d+2}$ for $|x-y| \gg 1$. The main result of the current paper implies that if $a<1$ then screening occurs in this sense provided we make the approximation $\left\langle\sin \left[\nabla_{i} \phi(x)\right] \sin \left[\nabla_{j} \phi(y)\right]\right\rangle \simeq$ $\left\langle\nabla_{i} \phi(x) \nabla_{j} \phi(y)\right\rangle$.

We state our result in terms of the Green's function for the homogenized constant coefficient elliptic PDE

$$
-\nabla \mathbf{a}_{\text {hom }} \nabla u_{\text {hom }}(x)=f(x), \quad x \in \mathbf{R}^{d},
$$

associated with the massless measure (1.1), which was obtained by Naddaf and Spencer [36].

Theorem 1.1. Let $V: \mathbf{R}^{d} \rightarrow \mathbf{R}$ be a $C^{3}$ function such that $V^{\prime \prime}(z), z \in \mathbf{R}^{d}$, is a diagonal $d \times d$ matrix, which satisfies the quadratic form inequality $\lambda I_{d} \leq$ $V^{\prime \prime}(z) \leq \Lambda I_{d}, z \in \mathbf{R}^{d}$, for some constants $\lambda, \Lambda>0$ and $\left\|V^{\prime \prime \prime}(\cdot)\right\|_{\infty}<\infty$. Let $G_{\mathbf{a}_{\mathrm{hom}}}(x), x \in \mathbf{R}^{d}$, be the Green's function for the Naddaf-Spencer PDE (1.6), and $\langle\cdot\rangle$ denote expectation for the $m \rightarrow 0$ massless measure of (1.1). Then for $d \geq 2$ there is a constant $\alpha>0$ depending only on $d$ and the ratio $\Lambda / \lambda$, and a constant $C$ depending only on $\left\|V^{\prime \prime \prime}(\cdot)\right\|_{\infty}, \Lambda, \lambda, d$ such that for $x \in \mathbf{Z}^{d}-\{0\}$,

$$
\begin{aligned}
\left|\langle\phi(x) \phi(0)\rangle-G_{\mathbf{a}_{\mathrm{hom}}}(x)\right| & \leq C /|x|^{d-2+\alpha}, \\
\left|\langle\nabla \phi(x) \phi(0)\rangle-\nabla G_{\mathbf{a}_{\mathrm{hom}}}(x)\right| & \leq C /|x|^{d-1+\alpha}, \\
\left|\left\langle\nabla \nabla^{*} \phi(x) \phi(0)\right\rangle-\nabla \nabla^{*} G_{\mathbf{a}_{\mathrm{hom}}}(x)\right| & \leq C /|x|^{d+\alpha} .
\end{aligned}
$$

REMARK 1. Note that both terms $\langle\phi(x) \phi(0)\rangle$ and $G_{\mathbf{a}_{\text {hom }}}(x)$ on the LHS of (1.7) are divergent in dimension $d=2$. However, the difference suitably defined is finite. The exponent $\alpha>0$ can be taken arbitrarily close to 1 by choosing $\lambda / \Lambda$ sufficiently close to 1 .

A correlation inequality like (1.7) which applies for the case of the dipole gas (1.5) was first proven by Gawedzki and Kupiainen [21] using a very complicated induction argument known as the renormalization group method. The renormalization group method they develop is very powerful and applies also to certain models in which the function $V(\cdot)$ is not uniformly convex. An example is the $(\nabla \phi)^{4}$ model for which $V(z)=|z|^{2} / 2+b \sum_{j=1}^{d}\left|z_{j}\right|^{4}$ with $b>0$. However the renormalization group method does not allow one to make a reasonable estimate on the value of $a$ in (1.5) for which the inequality (1.7) holds. In our case, the inequality is shown to hold for $a<1$. The induction argument 
of [21] was simplified and further developed in an influential paper of Brydges and Yau [6]. Using the method of [6], Dimock and Hurd [17] gave a new proof of (1.7) for a dipole gas model in $\mathbf{R}^{d}$ in which the potential is Coulomb with ultra-violet cutoff. They also obtained corresponding inequalities for the covariance of the variables $e^{i \phi(x)}, e^{-i \phi(0)}$ (Theorem 3 of [17]). More recently, Dimock [16] has taken a different renormalization group approach for the lattice dipole gas model, proving existence of the thermodynamic pressure. The inequalities (1.8), (1.9) are new in this paper even for the case of (1.5) with $a \ll 1$. In fact it seems unlikely to the authors that (1.9) can be proven using the renormalization group method. The reason is that the proof of (1.9) unlike the proof of (1.7), (1.8) — uses in an essential way a discrete version of the Nash-Moser Harnack inequality for solutions to parabolic PDE [15], [33].

The main technical tools we shall use to prove Theorem 1.1 are discrete versions of two classical theorems from the analysis of parabolic PDE. The first of these is a generalization by Jones [26] of the Calderon-Zygmund theorem to parabolic multipliers. Let $G(x, t), x \in \mathbf{R}^{d}, t>0$, be the Green's function for the heat equation

$$
\frac{\partial u(x, t)}{\partial t}=\Delta u(x, t), \quad x \in \mathbf{R}^{d}, t>0
$$

and extend $G$ to a function on $\mathbf{R}^{d+1}$ by setting $G(x, t)=0$ for $x \in \mathbf{R}^{d}, t<0$. We then define the convolution operator $T$ on functions $\psi: \mathbf{R}^{d+1} \rightarrow \mathbf{C}^{d}$ by

$$
T \psi(x, t)=\int_{\mathbf{R}^{d}} \int_{\mathbf{R}} \nabla \nabla^{*} G\left(x^{\prime}, t^{\prime}\right) \psi\left(x-x^{\prime}, t-t^{\prime}\right) d x^{\prime} d t^{\prime} .
$$

It is easy to see by going to Fourier variables that $T$ is a bounded operator on $L^{2}\left(\mathbf{R}^{d+1} ; \mathbf{C}^{d}\right)$. In fact, if $\hat{T}$ denotes the Fourier transformed operator $T$ then we have that

$$
\hat{T} \hat{\psi}(\xi, \theta)=\frac{\xi \xi^{*}}{i \theta+|\xi|^{2}} \hat{\psi}(\xi, \theta)
$$

where $\xi \in \mathbf{R}^{d}$ is the variable dual to $x \in \mathbf{R}^{d}$ and $\theta \in \mathbf{R}$ the variable dual to $t \in \mathbf{R}$. It follows from (1.12) that $T$ is bounded on $L^{2}\left(\mathbf{R}^{d+1} ; \mathbf{C}^{d}\right)$ with norm $\|T\|_{2}=1$. In [26] it is shown that for $1<p<\infty$ the operator $T$ is bounded on $L^{p}\left(\mathbf{R}^{d+1} ; \mathbf{C}^{d}\right)$ with norm $\|T\|_{p}<\infty$. Furthermore, one can see that $\lim _{p \rightarrow 2}\|T\|_{p}=1$. This convergence property of $\|T\|_{p}$ as $p \rightarrow 2$ plays a key role in our argument.

The second classical theorem we use is the Aronson inequality [1] for fundamental solutions to parabolic PDE. Let $\mathbf{a}: \mathbf{R}^{d} \times \mathbf{R}^{+} \rightarrow \mathbf{R}^{d(d+1) / 2}$ be a bounded function taking values in symmetric $d \times d$ matrices and satisfying the quadratic form inequality $\lambda I_{d} \leq \mathbf{a}(\cdot, \cdot) \leq \Lambda I_{d}$ for some constants $\lambda, \Lambda>0$. Aronson proved that if $G_{\mathbf{a}}(x, y, t), x, y \in \mathbf{R}^{d}, t>0$, is the fundamental solution 
to the PDE

$$
\frac{\partial u(x, t)}{\partial t}=\nabla \mathbf{a}(x, t) \nabla u(x, t), \quad x \in \mathbf{R}^{d}, t>0,
$$

then $G_{\mathbf{a}}$ satisfies an inequality

$$
G_{\mathbf{a}}(x, y, t) \leq C_{1} G\left(C_{2}(x-y), \Lambda t\right),
$$

where $G$ is the fundamental solution to the heat equation (1.10) and $C_{1}$, $C_{2}$ are positive constants depending only on $d, \Lambda / \lambda$. Aronson also proved a corresponding lower bound for $G_{\mathbf{a}}$, but this is not relevant for us.

The final key ingredient in the proof of Theorem 1.1 is the Poincaré inequality for the white noise process: Suppose $g(B(\cdot))$ is a suitably regular function of Brownian motion $B(t), t \geq 0$, and let $D_{\text {Mal }} g(t ; B(\cdot)), t>0$, denote the Malliavin derivative of $g$. Then one has (Theorem 5.4 of [8])

$$
\operatorname{Var}[g(B(\cdot))] \leq\left\langle\int_{0}^{\infty}\left|D_{\mathrm{Mal}} g(t ; B(\cdot))\right|^{2} d t\right\rangle .
$$

The proof of Theorem 1.1 proceeds by means of an identity obtained by Naddaf and Spencer [36] (proven rigorously in [22]), which relates the expectation $\langle\phi(x) \phi(0)\rangle$ for the massive measure (1.1) to an expectation of the Green's function for a parabolic partial difference equation with random coefficients. Let $(\Omega, \mathcal{F}, P)$ be a probability space equipped with measure preserving translation operators $\tau_{x, t}: \Omega \rightarrow \Omega, x \in \mathbf{Z}^{d}, t \in \mathbf{R}$, and $\mathbf{a}: \Omega \rightarrow \mathbf{R}^{d(d+1) / 2}$ be a function from $\Omega$ to the space of symmetric $d \times d$ matrices which satisfies the quadratic form inequality $\lambda I_{d} \leq \mathbf{a}(\omega) \leq \Lambda I_{d}, \omega \in \Omega$, for some positive constants $\Lambda, \lambda$. Solutions $u(x, t, \omega)$ to the random parabolic difference equation

$$
\frac{\partial u(x, t, \omega)}{\partial t}=-\nabla^{*} \mathbf{a}\left(\tau_{x, t} \omega\right) \nabla u(x, t, \omega), \quad x \in \mathbf{Z}^{d}, t \geq 0, \omega \in \Omega
$$

with initial data

$$
u(x, 0, \omega)=h(x), \quad x \in \mathbf{Z}^{d}, \omega \in \Omega,
$$

can be written in terms of a Green's function $G_{\mathbf{a}}$ as

$$
u(x, t, \omega)=\sum_{y \in \mathbf{Z}^{d}} G_{\mathbf{a}}(x, y, t, \omega) h(y), \quad x \in \mathbf{Z}^{d}, t>0 .
$$

Then one has that

$$
\langle\phi(x) \phi(0)\rangle=\int_{0}^{\infty} e^{-m^{2} t}\left\langle G_{\mathbf{a}}(x, 0, t, \cdot)\right\rangle d t
$$

for a particular probability space $(\Omega, \mathcal{F}, P)$ and function $\mathbf{a}: \Omega \rightarrow \mathbf{R}^{d(d+1) / 2}$. The notation $G_{\mathbf{a}}$ has been used in (1.14) and in (1.18) to refer to different Green's functions. We shall frequently use this notation for the Green's function of an evolution equation such as (1.13) or (1.16), which is associated with a symmetric matrix a. 
In [12], we studied expectations of the Green's function for random evolution equations (1.16), (1.17), and proved that for certain probability spaces $(\Omega, \mathcal{F}, P)$ they were well approximated on large length and time scales by the Green's function for the heat equation (1.10). The spaces $(\Omega, \mathcal{F}, P)$ were required to satisfy a strong independence condition for this result to hold (Theorem 1.3 of [12]). That is the variables $\mathbf{a}\left(\tau_{x, t} \cdot\right), x \in \mathbf{Z}^{d}, t \in \mathbf{R}$, needed to be approximately independent. In this paper, we shall prove that the results of Theorem 1.3 of [12] continue to hold for certain spaces $(\Omega, \mathcal{F}, P)$ in which the variables $\mathbf{a}\left(\tau_{x, t}\right), x \in \mathbf{Z}^{d}, t \in \mathbf{R}$, have long range correlations. The condition on $(\Omega, \mathcal{F}, P)$ is now given in terms of a uniform integrability assumption, which implies strong mixing of the variables $\mathbf{a}\left(\tau_{x, t}\right), x \in \mathbf{Z}^{d}, t \in \mathbf{R}$, but is much weaker than the approximate independence assumption. The space for which the identity (1.19) holds satisfies this uniform integrability assumption by virtue of a discrete version of Aronson's inequality (1.14). Theorem 1.1 then follows from (1.19) and our improvement (Theorem 2.2) of Theorem 1.3 of $[12]$.

\section{Rate of convergence in homogenization}

If the translation operators $\tau_{x, t}, x \in \mathbf{Z}^{d}, t \in \mathbf{R}$, are ergodic on $\Omega$ then solutions to the random evolution equation $(1.16),(1.17)$ converge under diffusive scaling to solutions of a constant coefficient homogenized equation. Thus suppose $f: \mathbf{R}^{d} \rightarrow \mathbf{R}$ is a $C^{\infty}$ function with compact support and for $\varepsilon$ satisfying $0<\varepsilon \leq 1$ set $h(x)=f(\varepsilon x), x \in \mathbf{Z}^{d}$, in (1.17), and let $u_{\varepsilon}(x, t, \omega)$ denote the corresponding solution to (1.16) with this initial data. It has been shown in [30], just assuming ergodicity of the translation operators, that $u_{\varepsilon}\left(x / \varepsilon, t / \varepsilon^{2}, \omega\right)$ converges in probability as $\varepsilon \rightarrow 0$ to a function $u_{\text {hom }}(x, t), x \in \mathbf{R}^{d}, t>0$, which is the solution to a constant coefficient parabolic PDE

$$
\frac{\partial u_{\mathrm{hom}}(x, t)}{\partial t}=\nabla \mathbf{a}_{\mathrm{hom}} \nabla u_{\mathrm{hom}}(x, t), \quad x \in \mathbf{R}^{d}, t>0
$$

with initial condition

$$
u_{\mathrm{hom}}(x, 0)=f(x), \quad x \in \mathbf{R}^{d} .
$$

If the matrix $\mathbf{a}(\cdot)$ of (1.16) satisfies the quadratic form inequality $\lambda I_{d} \leq \mathbf{a}(\cdot) \leq$ $\Lambda I_{d}$ for some $\lambda, \Lambda>0$, then the symmetric matrix $\mathbf{a}_{\text {hom }}$ in (2.1) also satisfies the inequality $\lambda I_{d} \leq \mathbf{a}_{\text {hom }} \leq \Lambda I_{d}$. Similar results under various ergodic type assumptions on $\Omega$ can be found in [3], [9], [18], [40]. In time-independent environments the corresponding results for elliptic equations in divergence form have been proven much earlier - see [28], [29], [39], [43].

In [12], we obtained a rate of convergence result for the homogenized limit, $\lim _{\varepsilon \rightarrow 0} u_{\varepsilon}\left(x / \varepsilon, t / \varepsilon^{2}, \omega\right)=u_{\text {hom }}(x, t)$. The corresponding problem for elliptic equations has been extensively studied, beginning with the seminal work of 
Yurinskii [42]. Recent papers on the subject have addressed the issue of obtaining optimal rates of convergence [23], [24], [31], and include results for fully non-linear elliptic equations [7]. Optimal estimates on variances of solutions have been obtained, but precise results on fluctuations analogous to the central limit theorem have been proven only in the case of one dimension [2]. In all these papers, one must make a quantitative strong mixing assumption on the environment $(\Omega, \mathcal{F}, P)$ in order to obtain a rate of convergence in homogenization. For the parabolic problem, the literature contains rather few results on rate of convergence in homogenization [34] (see also [25]), and for these the environment is fixed in time. In [12] as in [34], our results are restricted to obtaining a rate of convergence for the mean $\left\langle u_{\varepsilon}\left(x / \varepsilon, t / \varepsilon^{2}, \cdot\right)\right\rangle$ of the solution of $(1.16)$ to $u_{\text {hom }}(x, t)$. We were able to show that, for certain environments $(\Omega, \mathcal{F}, P)$ satisfying a quantitative strong mixing condition, there exists $\alpha>0$ depending only on $d, \Lambda / \lambda$ such that

$$
\sup _{x \in \varepsilon \mathbf{Z}^{d}, t>0}\left|\left\langle u_{\varepsilon}\left(x / \varepsilon, t / \varepsilon^{2}, \cdot\right)\right\rangle-u_{\text {hom }}(x, t)\right| \leq C \varepsilon^{\alpha} \quad \text { for } 0<\varepsilon \leq 1 .
$$

In [12], we followed the approach of Naddaf and Spencer [37] to the problem of obtaining rates of convergence in homogenization by formulating the quantitative strong mixing assumption on the environment as a Poincaré inequality. Specifically, consider a measure space $(\tilde{\Omega}, \tilde{\mathcal{F}})$ of time dependent vector fields $\tilde{\omega}: \mathbf{Z}^{d} \times \mathbf{R} \rightarrow \mathbf{R}^{k}$ with the property that the functions $t \rightarrow \tilde{\omega}(x, t), t \in \mathbf{R}$, are continuous for all $x \in \mathbf{Z}^{d}$ and each $\tilde{\omega}(x, t): \tilde{\Omega} \rightarrow \mathbf{R}^{k}$ is Borel measurable with respect to the $\sigma$-algebra $\tilde{\mathcal{F}}$. For a function $G: \tilde{\Omega} \rightarrow \mathbf{R}$ the gradient of $G$ is defined in a weak sense. Thus, if $h: \mathbf{Z}^{d} \times \mathbf{R} \rightarrow \mathbf{R}^{k}$ is continuous with compact support the directional derivative $d_{h} G(\tilde{\omega})$ of $G(\tilde{\omega})$ in the direction $h$ is defined as the limit

$$
d_{h} G(\tilde{\omega})=\lim _{\delta \rightarrow 0}[G(\tilde{\omega}+\delta h)-G(\tilde{\omega})] / \delta .
$$

The function $d_{\tilde{\omega}} G(\tilde{\omega}): \mathbf{Z}^{d} \times \mathbf{R} \rightarrow \mathbf{R}^{k}$ is then the gradient of $G$ at $\tilde{\omega}$ if it is Borel measurable and

$$
d_{h} G(\tilde{\omega})=\sum_{x \in \mathbf{Z}^{d}} \int_{-\infty}^{\infty} d t d_{\tilde{\omega}} G(x, t ; \tilde{\omega}) \cdot h(x, t)=\left[d_{\tilde{\omega}} G(\tilde{\omega}), h\right]
$$

for all continuous $h: \mathbf{Z}^{d} \times \mathbf{R} \rightarrow \mathbf{R}^{k}$ of compact support. In (2.5), we have denoted by $[\cdot, \cdot]$ the Euclidean inner product on $L^{2}\left(\mathbf{Z}^{d} \times \mathbf{R}, \mathbf{R}^{k}\right)$. Letting $\|\cdot\|_{2}$ denote the corresponding Euclidean norm, a probability measure $\tilde{P}$ on $(\tilde{\Omega}, \tilde{\mathcal{F}})$ is said to satisfy a Poincaré inequality if there is a constant $K_{\tilde{P}}>0$ such that

$$
\operatorname{Var}[G(\cdot)] \leq K_{\tilde{P}}\left\langle\left\|d_{\tilde{\omega}} G(\cdot ; \tilde{\omega})\right\|_{2}^{2}\right\rangle \quad \text { for all } C^{1} \text { functions } G: \tilde{\Omega} \rightarrow \mathbf{C} .
$$

If the translation invariant probability measure $\tilde{P}$ is Gaussian, then the measure is determined by the 2 -point correlation function $\Gamma: \mathbf{Z}^{d} \times \mathbf{R} \rightarrow \mathbf{R}^{k} \otimes$ 
$\mathbf{R}^{k}$ defined by $\Gamma(x, t)=\left\langle\tilde{\omega}(x, t) \tilde{\omega}(0,0)^{*}\right\rangle, x \in \mathbf{Z}^{d}, t \in \mathbf{R}$, where $\tilde{\omega}(\cdot, \cdot) \in \mathbf{R}^{k}$ is assumed to be a column vector and the superscript $*$ denotes adjoint. Defining the Fourier transform of a function $h: \mathbf{Z}^{d} \times \mathbf{R} \rightarrow \mathbf{C}$ by

$$
\hat{h}(\zeta, \theta)=\sum_{x \in \mathbf{Z}^{d}} \int_{-\infty}^{\infty} d t h(x, t) e^{i x \cdot \zeta+i t \theta}, \quad \zeta \in[-\pi, \pi]^{d}, \theta \in \mathbf{R},
$$

then the Poincaré inequality (2.6) holds if and only if $\hat{\Gamma} \in L^{\infty}\left([-\pi, \pi]^{d} \times \mathbf{R}\right)$. To see this, we observe that for a Gaussian measure $\tilde{P}$, the inequality (2.6) holds if and only if it holds for linear functions $G(\tilde{\omega})$ of the field $\tilde{\omega}$. Hence, (2.6) holds with constant $K_{\tilde{P}}$ if and only if

$$
\sum_{x, x^{\prime} \in \mathbf{Z}^{d}} \int_{-\infty}^{\infty} d t \int_{-\infty}^{\infty} d t^{\prime} f(x, t)^{*} \Gamma\left(x-x^{\prime}, t-t^{\prime}\right) f\left(x^{\prime}, t^{\prime}\right) \leq K_{\tilde{P}}\|f\|_{2}^{2}
$$

for all square integrable functions $f: \mathbf{Z}^{d} \times \mathbf{R} \rightarrow \mathbf{R}^{k}$. We conclude that if $\Gamma$ is integrable on $\mathbf{Z}^{d} \times \mathbf{R}$ then (2.6) holds, but it is unlikely to hold if $\Gamma$ is not integrable.

In the present paper, we shall prove rate of convergence results in homogenization of the parabolic PDE (1.16) for certain environments that include some Gaussian environments in which $\Gamma$ is not integrable. To do this, we extend the method introduced in [13] for elliptic PDE in divergence form to the parabolic case. The idea is to consider environments defined by $\mathbf{a}(\omega)=\tilde{\mathbf{a}}(\omega(0,0))$ where $\omega: \mathbf{Z}^{d} \times \mathbf{R} \rightarrow \mathbf{R}^{n}$ is a translation invariant function of $\tilde{\omega}: \mathbf{Z}^{d} \times \mathbf{R} \rightarrow \mathbf{R}^{k}$. The gradient of $\omega$ with respect to $\tilde{\omega}$ is assumed to satisfy a uniform integrability condition, and the probability space $(\tilde{\Omega}, \tilde{\mathcal{F}}, \tilde{P})$ for $\tilde{\omega}$ to satisfy the Poincaré inequality (2.6).

We define what we mean by the terms used in the previous paragraph. Let $(\Omega, \mathcal{F}, P)$ be the probability space for $\omega$ induced by the probability space $(\tilde{\Omega}, \tilde{\mathcal{F}}, \tilde{P})$ for $\tilde{\omega}$ and the functional dependence $\tilde{\omega} \rightarrow \omega$. Translation operators $\tau_{x, t}, x \in \mathbf{Z}^{d}, t \in \mathbf{R}$, on $\Omega$ are defined by $\tau_{x, t} \omega(z, s)=\omega(x+z, t+s), z \in \mathbf{Z}^{d}$, $s \in \mathbf{R}$, with a similar definition of translation on $\tilde{\Omega}$. The function $\tilde{\omega} \rightarrow \omega$, which we denote by $\omega(\cdot, \cdot, \tilde{\omega})$ is translation invariant if

$$
\tau_{x, t} \omega(\cdot, \cdot, \tilde{\omega})=\omega\left(\cdot, \cdot, \tau_{x, t} \tilde{\omega}\right) \quad \text { for } x \in \mathbf{Z}^{d}, t \in \mathbf{R}, \tilde{\omega} \in \tilde{\Omega} .
$$

Note that if $\omega$ is a linear translation invariant function of $\tilde{\omega}$ then $\omega$ is the convolution of some function $h: \mathbf{Z}^{d} \times \mathbf{R} \rightarrow \mathbf{R}^{n} \otimes \mathbf{R}^{k}$ from $\mathbf{Z}^{d} \times \mathbf{R}$ to $n \times k$ matrices with $\tilde{\omega}$,

$$
\begin{aligned}
& \omega(x, t, \tilde{\omega})=h * \tilde{\omega}(x, t)=\sum_{y \in \mathbf{Z}^{d}} \int_{-\infty}^{\infty} d s h(x-y, t-s) \tilde{\omega}(y, s), \\
& x \in \mathbf{Z}^{d}, t \in \mathbf{R} .
\end{aligned}
$$

For given $x \in \mathbf{Z}^{d}, t \in \mathbf{R}$ we use the notation of (2.5) to write the gradient of the function $\omega(x, t, \cdot): \tilde{\Omega} \rightarrow \mathbf{R}^{n}$ as $d_{\tilde{\omega}} \omega(z, s ; x, t, \tilde{\omega}), z \in \mathbf{Z}^{d}, s \in \mathbf{R}, \tilde{\omega} \in \tilde{\Omega}$. The 
uniform integrability condition is then that

$$
\sum_{x \in \mathbf{Z}^{d}} \int_{-\infty}^{\infty} d t\left[\sup _{\tilde{\omega} \in \tilde{\Omega}}\left|d_{\tilde{\omega}} \omega(0,0 ; x, t, \tilde{\omega})\right|\right]^{q} \leq\left(K_{\omega, q}\right)^{q}<\infty
$$

for some $q$ with $1 \leq q<2$. It follows from (2.10) that when $\omega$ is a linear function of $\tilde{\omega}$ the condition (2.11) is equivalent to the condition that the function $h$ in (2.10) is $q$ integrable.

In [12], we proved that $(2.3)$ holds in the case where $(\Omega, \mathcal{F}, P)$ is the stationary process associated with the massive $m>0$ invariant measure (1.1). The function $V: \mathbf{R}^{d} \rightarrow \mathbf{R}$ is assumed to be $C^{2}$ and satisfy the uniform convexity assumption $\lambda I_{d} \leq V^{\prime \prime}(\cdot) \leq \Lambda I_{d}$ for some $\lambda, \Lambda>0$. Consider functions $\phi: \mathbf{Z}^{d} \times \mathbf{R} \rightarrow \mathbf{R}$ which we denote as $\phi(x, t)$ where $x$ lies on the integer lattice $\mathbf{Z}^{d}$ and $t$ on the real line $\mathbf{R}$. Let $\Omega$ be the space of all such functions which have the property that for each $x \in \mathbf{Z}^{d}$ the function $t \rightarrow \phi(x, t)$ on $\mathbf{R}$ is continuous, and $\mathcal{F}$ be the Borel algebra generated by finite dimensional rectangles $\left\{\phi(\cdot, \cdot) \in \Omega:\left|\phi\left(x_{i}, t_{i}\right)-a_{i}\right|<r_{i}, i=1, \ldots, N\right\}$, where $\left(x_{i}, t_{i}\right) \in \mathbf{Z}^{d} \times \mathbf{R}, a_{i} \in \mathbf{R}$, $r_{i}>0, i=1, \ldots, N, N \geq 1$. For any $d \geq 1$ and $m>0$ one can define as in [10], [20] a unique ergodic translation invariant probability measure $P_{m}$ on $(\Omega, \mathcal{F})$ which depends on the function $V$ and $m$. In this measure the variables $\phi(x, t)$, $x \in \mathbf{Z}^{d}, t>0$, conditioned on the variables $\phi(x, 0), x \in \mathbf{Z}^{d}$, are determined as solutions of the infinite dimensional stochastic differential equation

$$
\begin{aligned}
d \phi(x, t)= & -\frac{\partial}{\partial \phi(x, t)} \sum_{x^{\prime} \in \mathbf{Z}^{d}} \frac{1}{2}\left\{V\left(\nabla \phi\left(x^{\prime}, t\right)\right)+m^{2} \phi\left(x^{\prime}, t\right)^{2} / 2\right\} d t \\
& +d B(x, t), \quad x \in \mathbf{Z}^{d}, t>0
\end{aligned}
$$

where $B(x, \cdot), x \in \mathbf{Z}^{d}$, are independent copies of Brownian motion. Formally the invariant measure for the Markov process (2.12) is the measure (1.1).

Hence, if the variables $\phi(x, 0), x \in \mathbf{Z}^{d}$, have distribution determined by (1.1), then $\phi(\cdot, t), t>0$, is a stationary process and so can be extended to all $t \in \mathbf{R}$ to yield a measure $P_{m}$ on $(\Omega, \mathcal{F})$. The probability space $\left(\Omega, \mathcal{F}, P_{m}\right)$ satisfies the Poincaré inequality (2.6) with constant $K_{P_{m}}=4 / m^{4}$ (Lemma 6.1 of [12]). In [12] we conclude from this that the inequality (2.3) holds provided $\mathbf{a}(\omega)=\tilde{\mathbf{a}}(\phi(0,0))$, where $\tilde{\mathbf{a}}: \mathbf{R} \rightarrow \mathbf{R}^{d(d+1) / 2}$ is assumed to be a $C^{1}$ function satisfying

$$
\|D \tilde{\mathbf{a}}(\cdot)\|_{\infty}<\infty, \quad \lambda I_{d} \leq \tilde{\mathbf{a}}(\cdot) \leq \Lambda I_{d} \text { for some } \lambda, \Lambda>0
$$

Let $(\tilde{\Omega}, \tilde{\mathcal{F}}, \tilde{P})$ be a probability space for which the Poincaré inequality (2.6) holds, and $\tilde{\omega} \rightarrow \omega$ a function which satisfies the translation invariant condition (2.9) and the uniform integrability condition (2.11) for some $q$ with $1 \leq q<2$. Our goal in the current paper is to show that the inequality $(2.3)$ holds for the environment $(\Omega, \mathcal{F}, P)$ of $\omega \in \Omega$ where $\mathbf{a}(\omega)=\tilde{\mathbf{a}}(\omega(0,0))$ and 
$\tilde{\mathbf{a}}: \mathbf{R} \rightarrow \mathbf{R}^{d(d+1) / 2}$ satisfies (2.13). Rather than attempt to formulate a general theorem for such environments, we shall only rigorously prove that $(2.3)$ holds for certain limits of the probability spaces $\left(\Omega, \mathcal{F}, P_{m}\right)$ defined by $(1.1)$, (2.12) as $m \rightarrow 0$. In Section 3, we indicate the generality of our argument by showing that the proof of Proposition 6.3 of [12] formally extends to the environment $(\Omega, \mathcal{F}, P)$.

From $(2.12)$, we see that the stationary process $\omega(\cdot, \cdot)=\phi(\cdot, \cdot)$ is a translation invariant function of the white noise stationary process $\tilde{\omega}(\cdot, \cdot)=d B(\cdot, \cdot)$. We see from (1.15) that the white noise process satisfies a Poincaré inequality (2.6) with $K_{\tilde{P}}=1$. Consider now the terminal value problem for the backwards in time parabolic PDE

$$
\begin{aligned}
\frac{\partial u(z, s)}{\partial s} & =\frac{1}{2} \nabla^{*} V^{\prime \prime}(\nabla \phi(z, s)) \nabla u(z, s), \quad s<t, z \in \mathbf{Z}^{d} \\
u(z, t) & =u_{0}(z), \quad z \in \mathbf{Z}^{d}
\end{aligned}
$$

The solution has the representation

$$
u(z, s)=\sum_{x \in \mathbf{Z}^{d}} G_{V^{\prime \prime}}(z, s ; x, t, \phi) u_{0}(x), \quad s<t, z \in \mathbf{Z}^{d}
$$

where $G_{V^{\prime \prime}}$ is the Green's function for (2.14). We see from equation (6.17) of [12] that the gradient of $\omega(x, t)=\phi(x, t), x \in \mathbf{Z}^{d}, t \in \mathbf{R}$, with respect to $\tilde{\omega}$ should be given by the formula

$$
\begin{aligned}
& d_{\tilde{\omega} \omega} \omega(z, s ; x, t, \tilde{\omega})=e^{-m^{2}(t-s) / 2} G_{V^{\prime \prime}}(z, s ; x, t, \phi) \quad \text { for } s<t, \\
& d_{\tilde{\omega} \omega} \omega(z, s ; x, t, \tilde{\omega})=0 \quad \text { for } s>t, z \in \mathbf{Z}^{d} .
\end{aligned}
$$

In [22] a discrete version of the Aronson inequality (1.14) was proven in the case when the diffusion matrix $V^{\prime \prime}(\cdot)$ for (2.14) is diagonal. In particular, it was shown that there is a positive constant $C$ depending only on $d, \Lambda / \lambda$ such that

$$
0<G_{V^{\prime \prime}}(z, s ; x, t, \phi) \leq \frac{C}{[\Lambda(t-s)+1]^{d / 2}} \exp \left[-\frac{|x-z|}{\sqrt{\Lambda(t-s)+1}}\right]
$$

Hence (2.16), (2.17) imply that the uniform integrability condition (2.11) holds for any $q$ with $q>1+2 / d$ and the bound on the RHS of (2.11) can be taken independent of $m$ as $m \rightarrow 0$. Hence, if $d \geq 3$ the condition (2.11) holds in the limit $m \rightarrow 0$ for some $q$ with $1 \leq q<2$.

It has been shown by Funaki and Spohn [20] (see also [10]) that if $d \geq 3$ then there is a unique limit as $m \rightarrow 0$ of the stationary process defined by (1.1), (2.12). In Section 4, we shall extend the rate of convergence results in homogenization of (1.16), (1.17) obtained in [12] for 
the massive field stationary process (1.1), (2.12) with $m>0$ to this massless $m \rightarrow 0$ stationary process. In particular, we prove the following theorem.

TheOREm 2.1. Let $V: \mathbf{R}^{d} \rightarrow \mathbf{R}$ be a $C^{2}$ function such that $V^{\prime \prime}(z), z \in \mathbf{R}^{d}$, is a diagonal $d \times d$ matrix which satisfies the quadratic form inequality $\lambda I_{d} \leq$ $V^{\prime \prime}(\cdot) \leq \Lambda I_{d}$ for some $\lambda, \Lambda>0$. Let $\tilde{\mathbf{a}}: \mathbf{R} \rightarrow \mathbf{R}^{d(d+1) / 2}$ be a $C^{1}$ function on $\mathbf{R}$ with values in the space of symmetric $d \times d$ matrices which satisfies (2.13). For $d \geq 3$ let $(\Omega, \mathcal{F}, P)$ be the probability space of the stationary process $\phi(\cdot, \cdot)$ determined by the limit as $m \rightarrow 0$ of the stationary process defined by (1.1), (2.12) and set $\mathbf{a}(\cdot)$ in (1.16) to be $\mathbf{a}(\phi)=\tilde{\mathbf{a}}(\phi(0,0)), \phi \in \Omega$. Let $f: \mathbf{R}^{d} \rightarrow \mathbf{R}$ be a $C^{\infty}$ function of compact support, $u_{\varepsilon}(x, t, \omega)$ the corresponding solution to (1.16), (1.17) with $h(x)=f(\varepsilon x), x \in \mathbf{Z}^{d}$, and $u_{\mathrm{hom}}(x, t), x \in \mathbf{R}^{d}, t>0$ the solution to (2.1), (2.2). Then there is a constant $\alpha>0$ depending only on $d$, $\Lambda / \lambda$ and a constant $C$ depending only on $d, \Lambda, \lambda,\|D \tilde{\mathbf{a}}(\cdot)\|_{\infty}, f(\cdot)$ such that (2.3) holds.

REMARK 2. The exponent $\alpha>0$ in (2.3) can be taken equal to 1 if $d \geq 5$ and the ratio $\lambda / \Lambda$ is sufficiently close to 1 . In [12] the matrix $V^{\prime \prime}(\cdot)$ is not required to be diagonal since we use the fact that the Poincaré inequality (2.6) holds for the massive field stationary process. In the Gaussian case where $V(\cdot)$ is quadratic $(2.3)$ also holds without the restriction that $V^{\prime \prime}(\cdot)$ be diagonal. This follows from the fact that a bound on the Green's function defined by (2.15) similar to (2.17) holds in this case. Another way of seeing it is to note that the field $\phi(\cdot, \cdot)$ is a linear translation invariant function of another field $\tilde{\omega}(\cdot, \cdot)$ as in $(2.10)$ with probability space which does satisfy a Poincaré inequality. This property of $\phi(\cdot, \cdot)$, being the convolution of a function with another field whose probability space satisfies a Poincaré inequality, does not seem to generalize to the case of uniformly convex $V(\cdot)$ which is not quadratic. One reason for this is that the measure for the time dependent stationary process $\phi(\cdot, \cdot)$ associated with $(2.12)$ appears to be $\log$ concave in $\phi(\cdot, \cdot)$ only in the Gaussian case when $V(\cdot)$ is quadratic (see Appendix A). In contrast, the invariant measure (1.1) for this process is easily seen to be $\log$ concave when $V(\cdot)$ is convex. Hence the Brascamp-Lieb inequality (1.3) implies that a Poincaré inequality holds for the gradient $\nabla \phi(\cdot)$ of the invariant measure field $\phi(\cdot)$ of $(1.1)$ if $V(\cdot)$ is uniformly convex (see $[14])$.

Parallel to [12], we also establish for the massless field stationary process point-wise convergence at large length scales of the averaged Green's function for the initial value problem (1.16), (1.17) to the homogenized Green's function for the initial value problem (2.1), (2.2). The averaged Green's function $G_{\mathbf{a}}(x, t), x \in \mathbf{Z}^{d}, t \geq 0$, for (1.16), (1.17) is defined by $G_{\mathbf{a}}(x, t)=\langle u(x, t, \cdot)\rangle$, 
where $h(\cdot)$ in (1.17) is the Kronecker delta function $h(x)=0$ if $x \neq 0$ and $h(0)=1$.

THEOREM 2.2. With the same environment as in the statement of Theorem 1.1, let $G_{\mathbf{a}_{\mathrm{hom}}}(x, t), x \in \mathbf{R}^{d}, t>0$, be the Green's function for the homogenized problem (2.1), (2.2). Then there are constants $\alpha, \gamma>0$ depending only on $d$ and the ratio $\Lambda / \lambda$ of the constants $\lambda, \Lambda$ of (2.13), and a constant $C$ depending only on $\|D \tilde{\mathbf{a}}(\cdot)\|_{\infty}, \Lambda, \lambda, d$ such that for $\Lambda t \geq 1$,

$$
\begin{aligned}
& \left|G_{\mathbf{a}}(x, t)-G_{\mathbf{a}_{\mathrm{hom}}}(x, t)\right| \\
& \quad \leq \frac{C}{[\Lambda t+1]^{(d+\alpha) / 2}} \exp \left[-\gamma \min \left\{|x|, \frac{|x|^{2}}{\Lambda t+1}\right\}\right] \\
& \left|\nabla G_{\mathbf{a}}(x, t)-\nabla G_{\mathbf{a}_{\mathrm{hom}}}(x, t)\right| \\
& \quad \leq \frac{C}{[\Lambda t+1]^{(d+1+\alpha) / 2}} \exp \left[-\gamma \min \left\{|x|, \frac{|x|^{2}}{\Lambda t+1}\right\}\right] \\
& \left|\nabla \nabla G_{\mathbf{a}}(x, t)-\nabla \nabla G_{\mathbf{a}_{\mathrm{hom}}}(x, t)\right| \\
& \quad \leq \frac{C}{[\Lambda t+1]^{(d+2+\alpha) / 2}} \exp \left[-\gamma \min \left\{|x|, \frac{|x|^{2}}{\Lambda t+1}\right\}\right] .
\end{aligned}
$$

Proof of Theorem 1.1. We identify the probability space $(\Omega, \mathcal{F}, P)$ and function $\mathbf{a}(\cdot)$ for which the identity (1.19) holds. The probability space is the space $\left(\Omega, \mathcal{F}, P_{m}\right)$ of stationary massive fields $\phi: \mathbf{Z}^{d} \times \mathbf{R} \rightarrow \mathbf{R}$ defined by (2.12). The function $\mathbf{a}(\cdot)$ is defined by $\mathbf{a}(\phi)=V^{\prime \prime}(\nabla \phi(0,0)), \phi \in \Omega$. To prove Theorem 1.1, we observe that the methods used to prove Theorem 2.2 for diffusion matrices $\mathbf{a}(\cdot)$ of the form $\mathbf{a}(\phi)=\tilde{\mathbf{a}}(\phi(0,0))$ can also be applied for diffusion matrices of the form $\mathbf{a}(\phi)=\tilde{\mathbf{a}}(\nabla \phi(0,0))$, where $\tilde{\mathbf{a}}: \mathbf{R}^{d} \rightarrow \mathbf{R}^{d /(d+1) / 2}$ is $C^{1}$ and satisfies (2.13). Instead of the bound (2.17), we use the inequality

$$
\left|\nabla_{x} G_{V^{\prime \prime}}(z, s ; x, t, \phi)\right| \leq \frac{C}{[\Lambda(t-s)+1]^{(d+\beta) / 2}} \exp \left[-\frac{|x-z|}{\sqrt{\Lambda(t-s)+1}}\right] .
$$

Evidently (2.21) with $\beta=0$ is a consequence of (2.17). This is sufficient to prove Theorem 1.1 when $d \geq 3$. To prove the theorem for $d=2$, we need to use the fact that $\beta$ can be chosen strictly positive depending only on $\lambda / \Lambda$. This follows from the Harnack inequality [15], [33]. Furthermore, $\beta$ can be chosen arbitrarily close to 1 provided $\lambda / \Lambda$ is sufficiently close to 1 . The result follows by letting $m \rightarrow 0$ in (1.19).

\section{Variance estimate on the solution to a PDE on $\Omega$}

We recall some definitions from [12]. For $\xi \in \mathbf{R}^{d}$ and $1 \leq j \leq d$, we define the $\xi$ derivative of a measurable function $\psi: \Omega \rightarrow \mathbf{C}$ in the $j$ direction by $\partial_{j, \xi}$, 
and its adjoint by $\partial_{j, \xi}^{*}$, where

$$
\begin{aligned}
& \partial_{j, \xi} \psi(\omega)=e^{-i \mathbf{e}_{j} \cdot \xi} \psi\left(\tau_{\mathbf{e}_{j}, 0} \omega\right)-\psi(\omega), \\
& \partial_{j, \xi}^{*} \psi(\omega)=e^{i \mathbf{e}_{j} \cdot \xi} \psi\left(\tau_{-\mathbf{e}_{j}, 0} \omega\right)-\psi(\omega) .
\end{aligned}
$$

We also define a $d$ dimensional column $\xi$ gradient operator $\partial_{\xi}$ by $\partial_{\xi}=$ $\left(\partial_{1, \xi}, \ldots, \partial_{d, \xi}\right)$, which has adjoint $\partial_{\xi}^{*}$ given by the row operator $\partial_{\xi}^{*}=$ $\left(\partial_{1, \xi}^{*}, \ldots, \partial_{d, \xi}^{*}\right)$. The time derivative of $\psi$ is defined by

$$
\partial \psi(\omega)=\lim _{\delta \rightarrow 0}\left[\psi\left(\tau_{0, \delta} \omega\right)-\psi(\omega)\right] / \delta .
$$

Let $\mathcal{H}(\Omega)$ be the Hilbert space of measurable functions $\Psi: \Omega \rightarrow \mathbf{C}^{d}$ with norm $\|\Psi\|_{\mathcal{H}(\Omega)}$ given by $\|\Psi\|_{\mathcal{H}(\Omega)}^{2}=\left\langle|\Psi(\cdot)|_{2}^{2}\right\rangle$, where $|\cdot|_{2}$ is the Euclidean norm on $\mathbf{C}^{d}$. Then there is a unique row vector solution $\Phi(\xi, \eta, \omega)=$ $\left(\Phi_{1}(\xi, \eta, \omega), \ldots, \Phi_{d}(\xi, \eta, \omega)\right)$ to the equation

$$
\begin{aligned}
& {[\eta+\partial] \Phi(\xi, \eta, \omega)+\partial_{\xi}^{*}\left[\mathbf{a}(\omega) \partial_{\xi} \Phi(\xi, \eta, \omega)\right]=-\partial_{\xi}^{*} \mathbf{a}(\omega),} \\
& \quad \eta>0, \xi \in \mathbf{R}^{d}, \omega \in \Omega,
\end{aligned}
$$

such that $\Phi(\xi, \eta, \cdot) v \in L^{2}(\Omega)$ for any $v \in \mathbf{C}^{d}$. The solution is in fact given by a convergent series expansion - see $(2.14)$ of [12]. Furthermore, $\Phi(\xi, \eta, \cdot) v \in$ $L^{2}(\Omega)$ satisfies the inequality

$$
\eta\|\Phi(\xi, \eta, \cdot) v\|_{L^{2}(\Omega)}^{2}+\lambda\left\|\partial_{\xi} \Phi(\xi, \eta, \cdot) v\right\|_{\mathcal{H}(\Omega)}^{2} \leq \Lambda^{2}|v|^{2} / \lambda .
$$

Letting $\mathcal{P}$ denote the projection orthogonal to the constant function, our generalization of Proposition 6.3 of [12] is as follows.

Proposition 3.1. Suppose $\mathbf{a}(\cdot)$ in (3.3) is given by $\mathbf{a}(\omega)=\tilde{\mathbf{a}}(\omega(0,0))$ where $\tilde{\mathbf{a}}: \mathbf{R}^{n} \rightarrow \mathbf{R}^{d(d+1) / 2}$ is a $C^{1} d \times d$ symmetric matrix valued function satisfying the quadratic form inequality (2.13) and $\|D \tilde{\mathbf{a}}(\cdot)\|_{\infty}<\infty$. The random field $\omega: \mathbf{Z}^{d} \times \mathbf{R} \rightarrow \mathbf{R}^{n}$ is a translation invariant function of a random field $\tilde{\omega}: \mathbf{Z}^{d} \times$ $\mathbf{R} \rightarrow \mathbf{R}^{k}$ which satisfies the uniform integrability condition (2.11) for some $q$ with $1 \leq q<2$. The probability space $(\tilde{\Omega}, \tilde{\mathcal{F}}, \tilde{P})$ of fields $\tilde{\omega}: \mathbf{Z}^{d} \times \mathbf{R} \rightarrow \mathbf{R}^{k}$ is assumed to satisfy the Poincaré inequality (2.6). Then there exists $q_{0}<2$ depending only on $d, \Lambda / \lambda$ such that if $q_{0} \leq q \leq 2$ and $g \in L^{p}\left(\mathbf{Z}^{d} \times \mathbf{R}, \mathbf{C}^{d} \otimes \mathbf{C}^{d}\right)$ with $p=2 q /(3 q-2)$ the inequality

$$
\begin{gathered}
\left\|\mathcal{P} \sum_{x \in \mathbf{Z}^{d}} \int_{-\infty}^{\infty} d t g(x, t) \partial_{\xi} \Phi\left(\xi, \eta, \tau_{x,-t} \cdot\right) v\right\|_{\mathcal{H}(\Omega)} \\
\leq \frac{C K_{\tilde{P}}^{1 / 2}\|D \tilde{\mathbf{a}}(\cdot)\|_{\infty}|v|}{\Lambda} K_{\omega, q}\|g\|_{p}, \quad v \in \mathbf{C}^{d},
\end{gathered}
$$

holds for a constant $C$ depending only on $d, n, k, \Lambda / \lambda, q_{0}$. 
Proof. From (2.6), we have that

$$
\begin{aligned}
& \left\|\mathcal{P} \sum_{x \in \mathbf{Z}^{d}} \int_{-\infty}^{\infty} d t g(x, t) \partial_{\xi} \Phi\left(\xi, \eta, \tau_{x,-t} \cdot\right) v\right\|_{\mathcal{H}(\Omega)}^{2} \\
& \leq K_{\tilde{P}} \sum_{z \in \mathbf{Z}^{d}} \int_{-\infty}^{\infty} d s\langle| \frac{\partial}{\partial \tilde{\omega}(z,-s)} \\
& \left.\quad \times\left.\sum_{x \in \mathbf{Z}^{d}} \int_{-\infty}^{\infty} d t g(x, t) \partial_{\xi} \Phi\left(\xi, \eta, \tau_{x,-t} \cdot\right) v\right|_{2} ^{2}\right\rangle .
\end{aligned}
$$

From the chain rule, we see that

$$
\begin{aligned}
& \frac{\partial}{\partial \tilde{\omega}(z,-s)} \partial_{\xi} \Phi\left(\xi, \eta, \tau_{x,-t} \cdot\right) v \\
& \quad=\sum_{y \in \mathbf{Z}^{d}} \int_{-\infty}^{\infty} d t^{\prime}\left[\frac{\partial}{\partial \omega\left(y, t^{\prime}\right)} \partial_{\xi} \Phi\left(\xi, \eta, \tau_{x,-t^{*}}\right) v\right] \cdot d_{\tilde{\omega}} \omega\left(z,-s ; y, t^{\prime}, \tilde{\omega}\right) .
\end{aligned}
$$

Next, we do a translation of the functions on the RHS of (3.7). Translation of a function $G: \tilde{\Omega} \rightarrow \mathbf{C}$ through $(x, t) \in \mathbf{Z}^{d} \times \mathbf{R}$ is defined by $\tau_{x, t} G(\tilde{\omega})=$ $G\left(\tau_{x, t} \tilde{\omega}\right), \tilde{\omega} \in \tilde{\Omega}$. For a function $G: \Omega \rightarrow \mathbf{C}$ there are two possible notions of translation through $(x, t)$, the first being given by $\tau_{x, t} G(\omega)=G\left(\tau_{x, t} \omega\right), \omega \in \Omega$. Since $\omega$ is a function of $\tilde{\omega} \in \tilde{\Omega}$ we can also define translation through $(x, t)$ by regarding $G: \Omega \rightarrow \mathbf{C}$ as a function on $\tilde{\Omega}$ and doing the translation on $\tilde{\Omega}$. It follows from the translation invariance property (2.9) that both of these notions are the same. Now using the translation invariance of the probability measure $\tilde{P}$ on $\tilde{\Omega}$ we conclude from $(3.6)$, (3.7) that

$$
\begin{aligned}
& \left\|\sum_{x \in \mathbf{Z}^{d}} \int_{-\infty}^{\infty} d t g(x, t) \partial_{\xi} \Phi\left(\xi, \eta, \tau_{x,-t^{*}}\right) v\right\|_{\mathcal{H}(\Omega)}^{2} \\
& \leq K_{\tilde{P}} \sum_{z \in \mathbf{Z}^{d}} \int_{-\infty}^{\infty} d s\langle| \sum_{x \in \mathbf{Z}^{d}} \int_{-\infty}^{\infty} d t g(x, t) \sum_{y \in \mathbf{Z}^{d}} \int_{-\infty}^{\infty} d t^{\prime} \\
& \left.\quad \times\left.\left[\tau_{-z, s} \frac{\partial}{\partial \omega\left(y, t^{\prime}\right)} \partial_{\xi} \Phi\left(\xi, \eta, \tau_{x,-t^{*}}\right) v\right] \cdot \tau_{-z, s} d_{\tilde{\omega}} \omega\left(z,-s ; y, t^{\prime}, \tilde{\omega}\right)\right|_{2} ^{2}\right\rangle .
\end{aligned}
$$

We define a function $u: \mathbf{Z}^{d} \times \mathbf{R} \times \tilde{\Omega} \rightarrow \mathbf{C}^{k}$ by

$$
\begin{aligned}
u(z, s, \tilde{\omega})= & e^{-i z \cdot \xi} \sum_{y \in \mathbf{Z}^{d}} \int_{-\infty}^{\infty} d t^{\prime} \\
& \times\left[d_{\omega} \Phi\left(y, t^{\prime} ; \xi, \eta, \tau_{z,-s} \omega\right) v\right] \cdot d_{\tilde{\omega}} \omega\left(0,0 ; y+z, t^{\prime}-s, \tilde{\omega}\right),
\end{aligned}
$$


where $d_{\omega} \Phi(\cdot ; \xi, \eta, \omega) v: \mathbf{Z}^{d} \times \mathbf{R} \rightarrow \mathbf{C}^{n}$ is the gradient of $\Phi(\xi, \eta, \omega) v$ with respect to $\omega \in \Omega$. Observe now from (2.9) that

$$
\begin{aligned}
d_{\tilde{\omega}} \omega\left(z,-s ; y, t^{\prime}, \tilde{\omega}\right) & =\frac{\partial}{\partial \tilde{\omega}(z,-s)} \omega\left(y, t^{\prime}, \tilde{\omega}\right) \\
& =\frac{\partial}{\partial \tilde{\omega}(z,-s)} \omega\left(y-z, t^{\prime}+s, \tau_{z,-s} \tilde{\omega}\right) \\
& =d_{\tilde{\omega}} \omega\left(0,0 ; y-z, t^{\prime}+s, \tau_{z,-s} \tilde{\omega}\right) .
\end{aligned}
$$

Hence, we have that

$$
\begin{aligned}
& e^{i(z-x) \cdot \xi} \sum_{y \in \mathbf{Z}^{d}} \int_{-\infty}^{\infty} d t^{\prime} \\
& \quad \times\left[\tau_{-z, s} \frac{\partial}{\partial \omega\left(y, t^{\prime}\right)} \Phi\left(\xi, \eta, \tau_{x,-t} \omega\right) v\right] \cdot \tau_{-z, s} d_{\tilde{\omega}} \omega\left(z,-s ; y, t^{\prime}, \tilde{\omega}\right) \\
&=e^{i(z-x) \cdot \xi} \sum_{y \in \mathbf{Z}^{d}} \int_{-\infty}^{\infty} d t^{\prime} \\
& \quad \times\left[d_{\omega} \Phi\left(y-x, t^{\prime}+t ; \xi, \eta, \tau_{x-z, s-t} \omega\right) v\right] \cdot d_{\tilde{\omega}} \omega\left(0,0 ; y-z, t^{\prime}+s, \tilde{\omega}\right) \\
&=e^{i(z-x) \cdot \xi \sum_{y \in \mathbf{Z}^{d}} \int_{-\infty}^{\infty} d t^{\prime}} \\
& \quad \times\left[d_{\omega} \Phi\left(y, t^{\prime} ; \xi, \eta, \tau_{x-z, s-t} \omega\right) v\right] \cdot d_{\tilde{\omega}} \omega\left(0,0 ; y+x-z, t^{\prime}+s-t, \tilde{\omega}\right) \\
&= u(x-z, t-s, \tilde{\omega}) .
\end{aligned}
$$

It follows from (3.11) that (3.8) can be rewritten as

$$
\begin{aligned}
& \left\|\mathcal{P} \sum_{x \in \mathbf{Z}^{d}} \int_{-\infty}^{\infty} d t g(x, t) \partial_{\xi} \Phi\left(\xi, \eta, \tau_{x,-t} \cdot\right) v\right\|_{\mathcal{H}(\Omega)}^{2} \\
& \leq K_{\tilde{P}} \sum_{z \in \mathbf{Z}^{d}} \int_{-\infty}^{\infty} d s \\
& \quad \times\left\langle\left|\sum_{x \in \mathbf{Z}^{d}} \int_{-\infty}^{\infty} d t g(x, t) e^{i(x-z) \cdot \xi} \nabla u(x-z, t-s, \cdot)\right|_{2}^{2}\right\rangle .
\end{aligned}
$$

In [12], we defined the $\xi$ derivative of a measurable function $\psi: \mathbf{Z}^{d} \times \mathbf{R} \times$ $\Omega \rightarrow \mathbf{C}$ in the $j$ direction by $D_{j, \xi}$, and its adjoint by $D_{j, \xi}^{*}$, where

$$
\begin{aligned}
D_{j, \xi} \psi(x, t ; \omega) & =e^{-i \mathbf{e}_{j} \cdot \xi} \psi\left(x-\mathbf{e}_{j}, t ; \tau_{\mathbf{e}_{j}} \omega\right)-\psi(x, t ; \omega), \\
D_{j, \xi}^{*} \psi(x, \omega) & =e^{i \mathbf{e}_{j} \cdot \xi} \psi\left(x+\mathbf{e}_{j}, t ; \tau_{-\mathbf{e}_{j}} \omega\right)-\psi(x, t ; \omega) .
\end{aligned}
$$

The corresponding $d$ dimensional column $\xi$ gradient operator $D_{\xi}$ is then given by $D_{\xi}=\left(D_{1, \xi}, \ldots, D_{d, \xi}\right)$, and it has adjoint $D_{\xi}^{*}$ given by the row operator 
$D_{\xi}^{*}=\left(D_{1, \xi}^{*}, \ldots, D_{d, \xi}^{*}\right)$. We also defined the time derivative $D_{0}$ of $\psi: \mathbf{Z}^{d} \times \mathbf{R} \times$ $\Omega \rightarrow \mathbf{C}$ by

$$
D_{0} \psi(x, t ; \omega)=\lim _{\delta \rightarrow 0}\left[\psi\left(x, t-\delta ; \tau_{0, \delta} \omega\right)-\psi(x, t ; \omega)\right] / \delta .
$$

Note that (3.13), (3.14) are generalizations of (3.1), (3.2) to functions which depend on $x \in \mathbf{Z}^{d}, t \in \mathbf{R}$ as well as $\omega \in \Omega$. We see from (3.1), (3.2) that these operators satisfy the identities

$$
\begin{array}{cl}
\frac{\partial}{\partial \omega(y, t)} \partial_{\xi} \psi(\omega)=D_{\xi} d_{\omega} \psi(y, t ; \omega), & y \in \mathbf{Z}^{d}, t \in \mathbf{R}, \omega \in \Omega, \\
\frac{\partial}{\partial \omega(y, t)} \partial \psi(\omega)=D_{0} d_{\omega} \psi(y, t ; \omega), & y \in \mathbf{Z}^{d}, t \in \mathbf{R}, \omega \in \Omega
\end{array}
$$

for differentiable functions $\psi: \Omega \rightarrow$ C. A similar relationship holds for the adjoints $\partial_{\xi}^{*}, D_{\xi}^{*}$. Hence on taking the gradient of equation (3.3) with respect to $\omega(\cdot)$, we conclude from (3.15) that

$$
\begin{aligned}
& {\left[\eta+D_{0}\right] d_{\omega} \Phi\left(y, t^{\prime} ; \xi, \eta, \omega\right) v+D_{\xi}^{*}\left[\tilde{\mathbf{a}}(\omega(0,0)) D_{\xi} d_{\omega} \Phi\left(y, t^{\prime} ; \xi, \eta, \omega\right)\right] v} \\
& =-D_{\xi}^{*}\left[\delta\left(y, t^{\prime}\right) D \tilde{\mathbf{a}}(\omega(0,0))\left\{v+\partial_{\xi} \Phi(\xi, \eta, \omega) v\right\}\right] \\
& \quad \text { for } y \in \mathbf{Z}^{d}, t^{\prime} \in \mathbf{R}, \omega \in \Omega .
\end{aligned}
$$

Evidently (3.16) holds with $\omega \in \Omega$ replaced by $\tau_{z,-s} \omega$ for any $z \in \mathbf{Z}^{d}$, $s \in \mathbf{R}$. We now multiply (3.16) with $\tau_{z,-s} \omega$ in place of $\omega$ on the right by $e^{-i z \cdot \xi} d_{\tilde{\omega}} \omega\left(0,0 ; y+z, t^{\prime}-s, \tilde{\omega}\right)$, sum with respect to $y \in \mathbf{Z}^{d}$ and integrate with respect to $t^{\prime} \in \mathbf{R}$. It then follows from (3.9), (3.16) that

$$
\begin{aligned}
& \eta u(z, s, \tilde{\omega})-\frac{\partial u(z, s, \tilde{\omega})}{\partial s}+\nabla^{*}[\tilde{\mathbf{a}}(\omega(z,-s)) \nabla u(z, s, \tilde{\omega})] \\
& \quad=-\nabla^{*} f(z, s, \tilde{\omega}),
\end{aligned}
$$

where the function $f: \mathbf{Z}^{d} \times \mathbf{R} \times \tilde{\Omega} \rightarrow \mathbf{C}^{d} \otimes \mathbf{C}^{k}$ is given by the formula

$$
\begin{aligned}
f(z, s, \tilde{\omega})= & D \tilde{\mathbf{a}}(\omega(z,-s))\left\{v+\partial_{\xi} \Phi\left(\xi, \eta, \tau_{z,-s} \omega\right) v\right\} \\
& \times e^{-i z \cdot \xi} d_{\tilde{\omega} \omega} \omega(0,0 ; z,-s, \tilde{\omega}) .
\end{aligned}
$$

For any $1<q<\infty$ we consider the function $f$ as a mapping $f: \mathbf{Z}^{d} \times \mathbf{R} \rightarrow$ $L^{2}\left(\tilde{\Omega}, \mathbf{C}^{d} \otimes \mathbf{C}^{k}\right)$ with norm defined by

$$
\|f\|_{q}^{q}=\sum_{y \in \mathbf{Z}^{d}} \int_{-\infty}^{\infty} d t^{\prime}\left\|f\left(y, t^{\prime}, \cdot\right)\right\|_{2}^{q},
$$

where $\left\|f\left(y, t^{\prime}, \cdot\right)\right\|_{2}$ is the norm of $f\left(y, t^{\prime} \cdot\right) \in L^{2}\left(\tilde{\Omega}, \mathbf{C}^{d} \otimes \mathbf{C}^{k}\right)$. Now from (3.4) it follows that $\partial_{\xi} \Phi(\xi, \eta, \cdot) v \in \mathcal{H}(\Omega)$ and $\left\|\partial_{\xi} \Phi(\xi, \eta, \cdot) v\right\|_{\mathcal{H}(\Omega)} \leq \Lambda|v| / \lambda$. Hence if the inequality (2.11) holds then the function $f$ is in $L^{q}\left(\mathbf{Z}^{d} \times \mathbf{R}, L^{2}\left(\tilde{\Omega}, \mathbf{C}^{d} \otimes\right.\right.$ $\left.\left.\mathbf{C}^{k}\right)\right)$ and $\|f\|_{q} \leq\|D \tilde{\mathbf{a}}(\cdot)\|_{\infty}(1+\Lambda / \lambda)|v| K_{\omega, q}$. We see from (3.17) that in the case $q=2$ then $\nabla u$ is also in $L^{2}\left(\mathbf{Z}^{d} \times \mathbf{R}, L^{2}\left(\tilde{\Omega}, \mathbf{C}^{d} \otimes \mathbf{C}^{k}\right)\right)$ and $\|\nabla u\|_{2} \leq\|f\|_{2} / \lambda$. It follows now from (3.12) that (3.5) holds with $q=2$ and $p=1$. 
To prove the inequality for some $p>1$, we use the parabolic version of Meyer's theorem [32]. We note that just as the Calderon-Zygmund theorem applies to functions with range in a Hilbert space [41], Jone's theorem for parabolic multipliers [26] also applies to functions with range in a Hilbert space (see Lemma 5.2 of [12]). We conclude that there exists $q_{0}$ depending only on $d, \Lambda / \lambda$ with $1<q_{0}<2$ such that if $\|f\|_{q}<\infty$ for any $q$ satisfying $q_{0} \leq q \leq 2$ then $\|\nabla u\|_{q} \leq 2\|f\|_{q} / \lambda$. Assume now that (2.11) holds for some $q$ in the interval $q_{0} \leq q \leq 2$. Then by Young's inequality for convolutions we see from (3.12) that (3.5) holds with $p=2 q /(3 q-2)$.

\section{Proof of Theorem 2.1 and Theorem 2.2}

The basic approach of [12] is to use the fact that the solution to (1.16) can be expressed by a Fourier inversion formula. For $\eta \in \mathbf{C}$, denote its real part by $\Re \eta \in \mathbf{R}$ and its imaginary part by $\Im \eta \in \mathbf{R}$ so that $\eta=\Re \eta+i \Im \eta$, and similarly denote the real and imaginary parts of $\xi \in \mathbf{C}^{d}$ by $\Re \xi, \Im \xi \in \mathbf{R}^{d}$ whence $\xi=\Re \xi+i \Im \xi$. We consider solutions to the equation

$$
\begin{aligned}
& {[\eta+\partial] \Phi(\xi, \eta, \omega)+\mathcal{P} \partial_{\xi}^{*} \mathbf{a}(\omega) \partial_{\xi} \Phi(\xi, \eta, \omega)=-\mathcal{P} \partial_{\xi}^{*} \mathbf{a}(\omega),} \\
& \quad \Re \eta>0, \xi \in \mathbf{R}^{d}, \omega \in \Omega .
\end{aligned}
$$

As with (3.3) there exists a unique solution to $(4.1)$ such that $\Phi(\xi, \eta, \cdot) v \in$ $L^{2}(\Omega)$ for any $v \in \mathbf{C}^{d}$. Furthermore, $\Phi(\xi, \eta, \cdot) v \in L^{2}(\Omega)$ satisfies the inequality

$$
\Re \eta\|\Phi(\xi, \eta, \cdot) v\|_{L^{2}(\Omega)}^{2}+\lambda\left\|\partial_{\xi} \Phi(\xi, \eta, \cdot) v\right\|_{\mathcal{H}(\Omega)}^{2} \leq \Lambda^{2}|v|^{2} / \lambda .
$$

If $\xi=0$, the solution $\Phi(\xi, \eta, \omega)$ to $(3.3)$ has zero mean so $\langle\Phi(0, \eta, \cdot)\rangle=0$. Hence, the solutions to (3.3), (4.1) coincide if $\xi=0$ but are in general different. For $\xi \in \mathbf{R}^{d}$ and $\eta \in \mathbf{C}$ with $\Re \eta>0$ let $e(\xi) \in \mathbf{C}^{d}$ be the vector $e(\xi)=\partial_{\xi} 1$ and $q(\xi, \eta)$ be the $d \times d$ matrix

$$
q(\xi, \eta)=\langle\mathbf{a}(\cdot)\rangle+\left\langle\mathbf{a}(\cdot) \partial_{\xi} \Phi(\xi, \eta, \cdot)\right\rangle
$$

where $\Phi(\xi, \eta, \omega)$ is the solution to (4.1). From equation (2.42) of [12], we have that the solution to $(1.16),(1.17)$ is given by the formula

$$
\begin{aligned}
u(x, t, \omega)= & \frac{1}{(2 \pi)^{d+1}} \int_{[-\pi, \pi]^{d}} \int_{-\infty}^{\infty} \frac{\hat{h}(\xi) e^{-i \xi \cdot x+\eta t}}{\eta+e(\xi)^{*} q(\xi, \eta) e(\xi)} \\
& \times\left[1+\Phi\left(\xi, \eta, \tau_{x, t} \omega\right) e(\xi)\right] d[\Im \eta] d \xi .
\end{aligned}
$$

If the environment $(\Omega, \mathcal{F}, P)$ is ergodic then the $\operatorname{limit}_{\lim _{\eta \rightarrow 0}} q(0, \eta)=\mathbf{a}_{\text {hom }}$ exists (see Lemma 2.5 of [11] for a proof in the discrete time case), and $\mathbf{a}_{\text {hom }}$ is the diffusion matrix for the homogenized equation (2.1). Let $\hat{G}_{\mathbf{a}}(\xi, \eta)$, $\xi \in[-\pi, \pi]^{d}, \Re \eta>0$, be the Fourier-Laplace transform of the averaged Green's function $G_{\mathbf{a}}(x, t), x \in \mathbf{Z}^{d}, t \geq 0$, for (1.16), (1.17) defined by

$$
\hat{G}_{\mathbf{a}}(\xi, \eta)=\int_{0}^{\infty} d t \sum_{x \in \mathbf{Z}^{d}} G_{\mathbf{a}}(x, t) \exp [i x . \xi-\eta t] .
$$


It follows from (4.4) that $\hat{G}_{\mathbf{a}}(\xi, \eta)$ is given by the formula

$$
\hat{G}_{\mathbf{a}}(\xi, \eta)=1 /\left[\eta+e(\xi)^{*} q(\xi, \eta) e(\xi)\right] \quad \text { for } \xi \in[-\pi, \pi]^{d}, \Re \eta>0 .
$$

In [12] it was shown that Theorem 2.1 and Theorem 2.2 are consequences of the following.

Hypothesis 4.1. For $\xi \in \mathbf{C}^{d}, \eta \in \mathbf{C}$ there exist positive constants $C_{1}$ and $\alpha \leq 1$ depending only on $d$ and $\Lambda / \lambda$, such the function $q(\xi, \eta), \xi \in \mathbf{R}^{d}, \Re \eta>0$, has an analytic continuation to the region $|\Im \xi|<C_{1} \sqrt{\Re \eta / \Lambda}, 0<\Re \eta<\Lambda$, and

$$
\begin{aligned}
& \left\|q\left(\xi^{\prime}, \eta^{\prime}\right)-q(\xi, \eta)\right\| \leq C \Lambda\left[\left|\xi^{\prime}-\xi\right|^{\alpha}+\left|\left(\eta^{\prime}-\eta\right) / \Lambda\right|^{\alpha / 2}\right], \\
& 0<\Re \eta \leq \Re \eta^{\prime} \leq \Lambda, \xi^{\prime}, \xi \in \mathbf{C}^{d} \text { with }|\Im \xi|,\left|\Im \xi^{\prime}\right| \leq C_{1} \sqrt{\Re \eta / \Lambda},
\end{aligned}
$$

where $C$ is a constant depending on the environment and the function $\mathbf{a}(\cdot)$.

This follows from Theorem 4.1 and Theorem 4.2 of [12]. Note that these theorems have been stated for discrete time, whence the requirement $4 d \Lambda \leq 1$. In the case of continuous time, there is no need for this restriction on the value of $\Lambda$ (see remarks at the end of $\S 4$ of [12]). Here we shall prove that Hypothesis 4.1 holds for the massless field environment $(\Omega, \mathcal{F}, P)$ of Theorem 2.1. To do this, we recall some operators defined in [12]. For any $g \in \mathcal{H}(\Omega)$, let $\psi(\xi, \eta, \omega)$ be the solution to the equation

$$
\frac{1}{\Lambda}[\eta+\partial] \psi(\xi, \eta, \omega)+\partial_{\xi}^{*} \partial_{\xi} \psi(\xi, \eta, \omega)=\partial_{\xi}^{*} g(\omega), \quad \Re \eta>0, \xi \in \mathbf{R}^{d}, \omega \in \Omega .
$$

The operator $T_{\xi, \eta}$ on $\mathcal{H}(\Omega)$ is defined by $T_{\xi, \eta} g(\cdot)=\partial_{\xi} \psi(\xi, \eta, \cdot)$. Let $G(x, t)$, $x \in \mathbf{Z}^{d}, t>0$, be the solution to the initial value problem

$$
\begin{aligned}
\frac{\partial G(x, t)}{\partial t}+\nabla^{*} \nabla G(x, t) & =0, \quad x \in \mathbf{Z}^{d}, t>0, \\
G(x, 0) & =\delta(x), \quad x \in \mathbf{Z}^{d} .
\end{aligned}
$$

It is well known [35] that there exist positive constants $C, \gamma$ depending only on $d$ such that $G$ satisfies the inequality

$$
\begin{aligned}
& G(x, t)+(t+1)^{1 / 2}|\nabla G(x, t)|+(t+1)\left|\nabla \nabla^{*} G(x, t)\right| \\
& \quad \leq \frac{C}{[t+1]^{d / 2}} \exp \left[-\gamma \min \left\{|x|, \frac{|x|^{2}}{t+1}\right\}\right], \quad \text { for } x \in \mathbf{Z}^{d}, t \geq 0
\end{aligned}
$$

From (2.47) of [12], we have that the operator $T_{\xi, \eta}$ is also given by the formula

$$
T_{\xi, \eta} g(\omega)=\Lambda \int_{0}^{\infty} e^{-\eta t} d t \sum_{x \in \mathbf{Z}^{d}}\left\{\nabla \nabla^{*} G(x, \Lambda t)\right\}^{*} \exp [-i x . \xi] g\left(\tau_{x,-t} \omega\right) .
$$

It easily follows from (4.8) that $T_{\xi, \eta}$ is a bounded operator on $\mathcal{H}(\Omega)$ with $\left\|T_{\xi, \eta}\right\|_{\mathcal{H}(\Omega)} \leq 1$ provided $\xi \in \mathbf{R}^{d}, \Re \eta>0$. Furthermore by Lemma 2.1 of [12], the function $(\xi, \eta) \rightarrow T_{\xi, \eta}$ from $\mathbf{R}^{d} \times \mathbf{R}$ to the Banach space of bounded linear 
operators on $\mathcal{H}(\Omega)$ has an analytic continuation to a strip $0<\Re \eta<\Lambda$, $|\Im \xi|<$ $C \sqrt{\Re \eta / \Lambda}$ where $C$ is a constant depending only on $d$.

Let $\mathbf{b}$ be the $d \times d$ matrix valued function $\mathbf{b}(\omega)=I_{d}-\mathbf{a}(\omega) / \Lambda, \omega \in \Omega$, whence (2.13) implies the quadratic form inequality $0 \leq \mathbf{b}(\cdot) \leq(1-\lambda / \Lambda) I_{d}$. It follows from (4.3) - see (2.21) of [12] - that

$$
q(\xi, \eta)=\langle\mathbf{a}(\cdot)\rangle-\Lambda \sum_{m=1}^{\infty}\left\langle\mathbf{b}(\cdot)\left[\mathcal{P} T_{\xi, \eta} \mathbf{b}(\cdot)\right]^{m}\right\rangle .
$$

We consider $\xi \in \mathbf{C}^{d}, \eta \in \mathbf{C}$ with $\xi$ having fixed imaginary part, $\eta$ having fixed positive real part, and satisfying the conditions of Hypothesis 4.1. For $k=$ $1,2, \ldots$, we define an operator $T_{k, \Im \xi, \Re \eta}$ from functions $g: \mathbf{Z}^{d} \times \mathbf{R} \rightarrow \mathbf{C}^{d} \otimes \mathbf{C}^{d}$ to periodic functions $T_{k, \Im \xi, \Re \eta} g:[-\pi, \pi]^{d} \times \mathbf{R} \times \Omega \rightarrow \mathbf{C}^{d} \otimes \mathbf{C}^{d}$ by

$$
T_{k, \Im \xi, \Re \eta} g(\Re \xi, \Im \eta, \cdot)=\sum_{x \in \mathbf{Z}^{d}} \int_{-\infty}^{\infty} d t g(x, t) \tau_{x,-t} \mathcal{P} \mathbf{b}(\cdot)\left[\mathcal{P} T_{\xi, \eta} \mathbf{b}(\cdot)\right]^{k-1},
$$

where $\xi=\Re \xi+i \Im \xi, \eta=\Re \eta+i \Im \eta$ in (4.13). For $1 \leq p<\infty$ let $L^{p}\left(\mathbf{Z}^{d} \times \mathbf{R}\right.$, $\mathbf{C}^{d} \otimes \mathbf{C}^{d}$ ) be the Banach space of $d \times d$ matrix valued functions $g: \mathbf{Z}^{d} \times \mathbf{R} \rightarrow$ $\mathbf{C}^{d} \otimes \mathbf{C}^{d}$ with norm $\|g\|_{p}$ defined by

$$
\|g\|_{p}^{p}=\sup _{v \in \mathbf{C}^{d}:|v|=1} \sum_{x \in \mathbf{Z}^{d}} \int_{-\infty}^{\infty} d t|g(x, t) v|_{2}^{p},
$$

where $|g(x, t) v|_{2}$ is the Euclidean norm of the vector $g(x, t) v \in \mathbf{C}^{d}$. We similarly define the space $L^{\infty}\left([-\pi, \pi]^{d} \times \mathbf{R} \times \Omega, \mathbf{C}^{d} \otimes \mathbf{C}^{d}\right)$ of $d \times d$ matrix valued functions $g:[-\pi, \pi]^{d} \times \mathbf{R} \times \Omega \rightarrow \mathbf{C}^{d} \otimes \mathbf{C}^{d}$ with norm $\|g\|_{\infty}$ defined by

$$
\|g\|_{\infty}=\sup _{v \in \mathbf{C}^{d}:|v|=1}\left[\sup _{\zeta \in[-\pi, \pi]^{d}, \theta \in \mathbf{R}}\|g(\zeta, \theta, \cdot) v\|_{\mathcal{H}(\Omega)}\right] .
$$

Since $\left\|T_{\xi, \eta}\right\|_{\mathcal{H}(\Omega)} \leq 1$ if $\xi \in \mathbf{R}^{d}, \Re \eta>0$ it follows from (4.13), (4.14) that if $\Im \xi=0$ then $T_{k, \Im \xi, \Re \eta}$ is a bounded operator from $L^{1}\left(\mathbf{Z}^{d} \times \mathbf{R}, \mathbf{C}^{d} \otimes \mathbf{C}^{d}\right)$ to $L^{\infty}\left([-\pi, \pi]^{d} \times \mathbf{R} \times \Omega, \mathbf{C}^{d} \otimes \mathbf{C}^{d}\right)$ with norm $\left\|T_{k, \Im \xi, \Re \eta}\right\|_{1, \infty} \leq(1-\lambda / \Lambda)^{k}$. In the next section we show that $T_{k, \Im \xi, \Re \eta}$ is a bounded operator from $L^{p}\left(\mathbf{Z}^{d} \times\right.$ $\left.\mathbf{R}, \mathbf{C}^{d} \otimes \mathbf{C}^{d}\right)$ to $L^{\infty}\left([-\pi, \pi]^{d} \times \mathbf{R} \times \Omega, \mathbf{C}^{d} \otimes \mathbf{C}^{d}\right)$ for some $p>1$ in the case of the environment of Theorem 2.1 and estimate its norm $\left\|T_{k, \Im \xi, \Re \eta}\right\|_{p, \infty}$. In particular, we prove the following lemma.

LEMMA 4.1. Let $(\Omega, \mathcal{F}, P)$ be an environment of massless fields $\phi: \mathbf{Z}^{d} \times$ $\mathbf{R} \rightarrow \mathbf{R}$ with $d \geq 3$, and $\tilde{\mathbf{a}}: \mathbf{R} \rightarrow \mathbf{R}^{d(d+1) / 2}$ be as in the statement of Theorem 2.1. Set $\mathbf{a}(\phi)=\tilde{\mathbf{a}}(\phi(0,0)), \phi \in \Omega$. Then there exists $p_{0}(\Lambda / \lambda)$ with $1<p_{0}(\Lambda / \lambda)<2$ depending only on $d$ and $\Lambda / \lambda$, and positive constants $C_{1}(\Lambda / \lambda), C_{2}(\Lambda / \lambda)$ depending only on $d$ and $\Lambda / \lambda$ such that for $0<\Re \eta<\Lambda$, 
$|\Im \xi|<C_{1}(\Lambda / \lambda) \sqrt{\Re \eta / \Lambda}$,

$$
\begin{aligned}
\left\|T_{k, \Im \xi, \Re \eta}\right\|_{p, \infty} \leq & \frac{C_{2}(\Lambda / \lambda) k\|D \tilde{\mathbf{a}}(\cdot)\|_{\infty}}{\Lambda^{5 / 2-1 / p}} \\
& \times(1-\lambda / \Lambda)^{(k-1) / 2}\left[1+C_{2}|\Im \xi|^{2} /(\Re \eta / \Lambda)\right]^{k-1}
\end{aligned}
$$

provided $1 \leq p \leq p_{0}(\Lambda / \lambda)$.

To complete this section, we show how Lemma 4.1 implies that Hypothesis 4.1 holds.

Proof of Hypothesis 4.1. We assume that $(\xi, \eta)$ and $\left(\xi^{\prime}, \eta^{\prime}\right)$ are as in the statement of Hypothesis 4.1. Let $g: \mathbf{Z}^{d} \times \mathbf{R} \rightarrow \mathbf{C}^{d} \otimes \mathbf{C}^{d}$ be the function defined by

$$
g(x, t)=\Lambda\left\{\nabla \nabla^{*} G(x, \Lambda t)\right\}^{*} e^{-i x \cdot \xi^{\prime}-\eta^{\prime} t}-\Lambda\left\{\nabla \nabla^{*} G(x, \Lambda t)\right\}^{*} e^{-i x \cdot \xi-\eta t}
$$

where the Green's function $G(\cdot, \cdot)$ is defined by $(4.9)$. We use Lemma 2.1 of [12], which also holds for continuous time translation operators (see remarks at the end of $\S 2$ of [12]). It then follows from (4.11), (4.12) that the constant $C_{1}>0$ in (4.7) can be chosen depending only on $d$ and $\Lambda / \lambda$ so that

$$
\begin{aligned}
& \left\|\left[q\left(\xi^{\prime}, \eta^{\prime}\right)-q(\xi, \eta)\right] v\right\| \leq C_{2} \Lambda \sum_{k=1}^{\infty}\left\|T_{k, \Im \xi, \Re \eta} g(\Re \xi, \Im \eta, \cdot) v\right\|_{\mathcal{H}(\Omega)} \\
& \quad \text { for }|\Im \xi|,\left|\Im \xi^{\prime}\right| \leq C_{1} \sqrt{\Re \eta / \Lambda},
\end{aligned}
$$

where $C_{2}$ is a constant depending only on $d, \Lambda / \lambda$. We can see from (4.10) that there is a constant $C_{1}$ depending only on $d$ such that if $|\Im \xi|,\left|\Im \xi^{\prime}\right|<C_{1} \sqrt{\Re \eta / \Lambda}$ then the function $g$ is in $L^{p}\left(\mathbf{Z}^{d} \times \mathbf{R}, \mathbf{C}^{d} \otimes \mathbf{C}^{d}\right)$ for any $p>1$. Furthermore if $0 \leq \alpha \leq 1$ and $p>(d+2) /(d+2-\alpha)$, then $\|g\|_{p}$ satisfies the inequality

$$
\|g\|_{p} \leq C_{p} \Lambda^{1-1 / p}\left[\left|\xi^{\prime}-\xi\right|^{\alpha}+\left|\left(\eta^{\prime}-\eta\right) / \Lambda\right|^{\alpha / 2}\right],
$$

where the constant $C_{p}$ depends only on $d, p$. The Hölder continuity (4.7) for sufficiently small $\alpha>0$ follows from (4.18), (4.19) and Lemma 4.1.

\section{Proof of Lemma 4.1}

In [12], we proved that the operator $T_{k, \Im \xi, \Re \eta}$ of (4.13) is for some $p$ in the range $1 \leq p \leq p_{0}(\Lambda / \lambda)$ a bounded operator from $L^{p}\left(\mathbf{Z}^{d} \times \mathbf{R}, \mathbf{C}^{d} \otimes \mathbf{C}^{d}\right)$ to $L^{\infty}\left([-\pi, \pi]^{d} \times \mathbf{R} \times \Omega, \mathbf{C}^{d} \otimes \mathbf{C}^{d}\right)$ if the environment $(\Omega, \mathcal{F}, P)$ is the stationary process for the SDE (2.12) with $m>0$. Here, we take an alternative approach to proving this result which will allow us to study the $m \rightarrow 0$ limit of $\left\|T_{k, \Im \xi, \Re \eta}\right\|_{p, \infty}$. We first establish an inequality for periodic fields $\phi: Q \rightarrow \mathbf{R}$ on cubes $Q \subset \mathbf{Z}^{d}$, and then show that we can let $Q \rightarrow \mathbf{Z}^{d}$ since our estimates are independent of $Q$. Let $L$ be an even integer and $Q=Q_{L}$ denote the lattice points of $\mathbf{Z}^{d}$ contained in the cube of length $L$ centered at the origin. 
In the following, we identify all points $x, y \in Q$ with $x-y=L \mathbf{e}_{k}$ for some $k$, $1 \leq k \leq d$.

As in [12], the Malliavin calculus [8], [38] is the main tool we use to prove Lemma 4.1. We assume that $V: \mathbf{R}^{d} \rightarrow \mathbf{R}$ is a $C^{2}$ uniformly convex function such that $\mathbf{a}(\cdot)=V^{\prime \prime}(\cdot)$ satisfies (2.13) and $m>0$. Letting $B(x, \cdot), x \in Q$, be independent copies of Brownian motion, then the SDE initial value problem

(5.1) $d \phi(x, t)$

$$
=-\frac{\partial}{\partial \phi(x, t)} \sum_{x^{\prime} \in Q} \frac{1}{2}\left\{V\left(\nabla \phi\left(x^{\prime}, t\right)\right)+m^{2} \phi\left(x^{\prime}, t\right)^{2} / 2\right\} d t+d B(x, t)
$$

for $x \in Q, t>0$, with $\phi(x, 0)=0$ for $x \in Q$,

has a unique periodic solution $\phi(x, t), x \in Q, t>0$, which is continuous in $t \geq 0$ with probability 1 . We denote the function $\phi$ corresponding to a particular realization $\tilde{\omega}$ of the white noise process $d B(\cdot, \cdot)$ as $\phi(\tilde{\omega})$. Let $\left(\Omega_{Q, \text { Mal }}, \mathcal{F}_{Q, \text { Mal }}, P_{Q, \text { Mal }}\right)$ be the Malliavin probability space associated with the Brownian motions $B(x, \cdot), x \in Q$. We denote the Malliavin derivative of a function $G: \Omega_{Q, \text { Mal }} \rightarrow \mathbf{C}$ at a point $\tilde{\omega} \in \Omega_{Q, \text { Mal }}$ by $D_{\text {Mal }} G(x, t ; \tilde{\omega}), x \in Q$, $t>0$. It is well known (see [8] Theorem 5.4) that the Poincaré inequality (2.6) holds for $\left(\Omega_{Q, \mathrm{Mal}}, \mathcal{F}_{Q, \mathrm{Mal}}, P_{Q, \mathrm{Mal}}\right)$ with constant $K_{\tilde{P}}=1$. Thus, we have that

$$
\operatorname{Var}[G(\cdot)] \leq\left\langle\left\|D_{\mathrm{Mal}} G(\cdot ; \tilde{\omega})\right\|_{2}^{2}\right\rangle_{\Omega_{Q, \mathrm{Mal}}}
$$

where $\|\cdot\|_{2}$ is the Euclidean norm in $L^{2}\left(Q \times \mathbf{R}^{+}\right)$.

Let $\phi: \mathbf{Z}^{d} \times \mathbf{R} \rightarrow \mathbf{R}$ be continuous and consider the terminal value problem for the backwards in time parabolic PDE

$$
\begin{aligned}
\frac{\partial u(y, s)}{\partial s} & =\frac{1}{2} \nabla^{*} V^{\prime \prime}(\nabla \phi(y, s)) \nabla u(y, s), \quad s<t, y \in \mathbf{Z}^{d} \\
u(y, t) & =u_{0}(y), \quad y \in \mathbf{Z}^{d}
\end{aligned}
$$

with solution

$$
u(y, s)=\sum_{x \in \mathbf{Z}^{d}} G(y, s ; x, t, \phi) u_{0}(x), \quad s \leq t, y \in \mathbf{Z}^{d} .
$$

It is easy to see that if $u_{0}(\cdot) \in L^{2}\left(\mathbf{Z}^{d}\right)$ then $u(\cdot, s) \in L^{2}\left(\mathbf{Z}^{d}\right)$ for $s \leq t$ and $\|u(\cdot, s)\|_{L^{2}\left(\mathbf{Z}^{d}\right)} \leq\left\|u_{0}(\cdot)\right\|_{L^{2}\left(\mathbf{Z}^{d}\right)}$. The function $G$ satisfies

$$
\sum_{x \in \mathbf{Z}^{d}} G(y, s ; x, t, \phi)=1, \quad y \in \mathbf{Z}^{d} ; \quad \sum_{y \in \mathbf{Z}^{d}} G(y, s ; x, t, \phi)=1, \quad x \in \mathbf{Z}^{d}
$$

and is non-negative if $V^{\prime \prime}(\cdot)$ is diagonal. In that case, the function $x \rightarrow$ $G(y, s ; x, t, \phi), x \in \mathbf{Z}^{d}$, is the pdf for the position at time $t$ of a continuous time random walk started at $y$ at time $s$. If $\phi: Q \times \mathbf{R} \rightarrow \mathbf{R}$ is periodic we can extend it to a periodic function $\phi: \mathbf{Z}^{d} \times \mathbf{R} \rightarrow \mathbf{R}$. Let $u_{0}: Q \rightarrow \mathbf{R}$ be periodic 
and extend it to a periodic function $u_{0}: \mathbf{Z}^{d} \rightarrow \mathbf{R}$. Then the solution to the periodic terminal value problem (5.3) is given by

$$
u(y, s)=\sum_{x \in Q} G_{Q}(y, s ; x, t, \phi) u_{0}(x), \quad s \leq t, y \in Q
$$

where $G_{Q}$ is the periodic Green's function

$$
G_{Q}(y, s ; x, t)=\sum_{n \in \mathbf{Z}^{d}} G(y, s ; x+L n, t, \phi), \quad s \leq t, x, y \in \mathbf{Z}^{d} .
$$

It was shown in [12] - see equation (6.17) - that the Malliavin derivative of $\phi(x, t, \tilde{\omega}), x \in Q, t>0$, is given by the formula

$$
\begin{aligned}
& D_{\mathrm{Mal}} \phi(y, s ; x, t, \tilde{\omega})=e^{-m^{2}(t-s) / 2} G_{Q}(y, s ; x, t, \phi(\tilde{\omega})) \quad \text { for } 0<s<t, \\
& D_{\mathrm{Mal}} \phi(y, s ; x, t, \tilde{\omega})=0 \quad \text { for } s>t, y \in Q .
\end{aligned}
$$

The solution to $(5.3)$ can be written in a perturbation expansion by setting $V^{\prime \prime}(z)=\Lambda\left[I_{d}-\tilde{\mathbf{b}}_{V}(z)\right], z \in \mathbf{R}^{d}$, where $0 \leq \tilde{\mathbf{b}}_{V}(\cdot) \leq(1-\lambda / \Lambda) I_{d}$ in the quadratic form sense. Then

$$
u(y, s)=\sum_{n=0}^{\infty} u_{n}(y, s), \quad y \in \mathbf{Z}^{d}, s<t
$$

where $u_{0}(y, s)$ is the solution to the terminal value problem

$$
\begin{aligned}
\frac{\partial u_{0}(y, s)}{\partial s} & =\frac{\Lambda}{2} \nabla^{*} \nabla u_{0}(y, s), \quad s<t, y \in \mathbf{Z}^{d}, \\
u_{0}(y, t) & =u_{0}(y), \quad y \in \mathbf{Z}^{d},
\end{aligned}
$$

and the $u_{n}(y, s), n=1,2, \ldots$, solutions to the terminal value problems

$$
\begin{aligned}
& \frac{\partial u_{n}(y, s)}{\partial s}=\frac{\Lambda}{2}\left[\nabla^{*} \nabla u_{n}(y, s)-\nabla^{*} \tilde{\mathbf{b}}_{V}(\nabla \phi(y, s)) \nabla u_{n-1}(y, s)\right], \\
& s<t, y \in \mathbf{Z}^{d}, \\
& u_{n}(y, t)=0, \quad y \in \mathbf{Z}^{d} .
\end{aligned}
$$

It follows from (4.9) that

$$
u_{0}(y, s)=\sum_{z \in \mathbf{Z}^{d}} G(y-z, \Lambda(t-s) / 2) u_{0}(z), \quad y \in \mathbf{Z}^{d}, s<t .
$$

Similarly we have that for $n \geq 1$,

$$
\begin{aligned}
u_{n}(z, r)= & \sum_{y \in \mathbf{Z}^{d}} \int_{0}^{t-r} d s \nabla G(y, \Lambda s / 2) \tilde{\mathbf{b}}_{V}(\nabla \phi(z+y, r+s)) \\
& \times \nabla u_{n-1}(z+y, r+s), \quad z \in \mathbf{Z}^{d}, r<t
\end{aligned}
$$


If we set $u_{0}$ in (5.3) to be given by $u_{0}(y)=\delta(y-x), y \in \mathbf{Z}^{d}$, then the perturbation expansion (5.9) yields a perturbation expansion for the Green's function,

$$
G(y, s ; x, t, \phi)=\sum_{n=0}^{\infty} G_{n}(y, s ; x, t, \phi),
$$

where the $G_{n}$ are multilinear in $\tilde{\mathbf{b}}_{V}$ of degree $n$. By choosing $u_{0}(y)=$ $\sum_{n \in \mathbf{Z}^{d}} \delta(y-x-n L), y \in \mathbf{Z}^{d}$, we obtain a similar perturbation expansion for the periodic Green's function

$$
G_{Q}(y, s ; x, t, \phi)=\sum_{n=0}^{\infty} G_{n, Q}(y, s ; x, t, \phi) .
$$

Next, we consider the inhomogeneous problem

$$
\begin{aligned}
\frac{\partial u(y, s)}{\partial s} & =\frac{1}{2} \nabla^{*} V^{\prime \prime}(\nabla \phi(y, s)) \nabla u(y, s)-f(y, s), \quad s \in \mathbf{R}, y \in \mathbf{Z}^{d}, \\
\lim _{s \rightarrow+\infty} u(y, s) & =0, \quad y \in \mathbf{Z}^{d} .
\end{aligned}
$$

Let $f: \mathbf{Z}^{d} \times \mathbf{R} \rightarrow \mathbf{C}$ be a continuous function such that $\int_{-\infty}^{\infty} d t\|f(\cdot, t)\|_{L^{2}\left(\mathbf{Z}^{d}\right)}<$ $\infty$. From Duhamel's formula we see that the solution to (5.16) is given by

$$
u(y, s)=\sum_{x \in \mathbf{Z}^{d}} \int_{s}^{\infty} d t G(y, s ; x, t, \phi) f(x, t), \quad s \in \mathbf{R}, y \in \mathbf{Z}^{d} .
$$

We can similarly consider the inhomogeneous periodic problem where $f: Q \times$ $\mathbf{R} \rightarrow \mathbf{C}$ is assumed periodic and we extend it to a periodic function $f: \mathbf{Z}^{d} \times$ $\mathbf{R} \rightarrow \mathbf{C}$. The solution to (5.16) is then periodic and is given by the formula

$$
u(y, s)=\sum_{x \in Q} \int_{s}^{\infty} d t G_{Q}(y, s ; x, t, \phi) f(x, t), \quad s \in \mathbf{R}, y \in Q .
$$

Lemma 5.1. Assume $g: \mathbf{Z}^{d} \times \mathbf{R} \rightarrow \mathbf{C}$ is in $L^{2}\left(\mathbf{Z}^{d} \times \mathbf{R}\right)$ and define for $m>0$ the function $v: \mathbf{Z}^{d} \times \mathbf{R} \rightarrow \mathbf{C}$ by

$$
v(y, s)=\sum_{x \in \mathbf{Z}^{d}} \int_{s}^{\infty} d t e^{-m^{2}(t-s) / 2} G(y, s ; x, t, \phi) g(x, t), \quad s \in \mathbf{R}, y \in \mathbf{Z}^{d} .
$$

Then $v$ is also in $L^{2}\left(\mathbf{Z}^{d} \times \mathbf{R}\right)$ and $\|v(\cdot, \cdot)\|_{L^{2}\left(\mathbf{Z}^{d} \times \mathbf{R}\right)} \leq 2 m^{-2}\|g(\cdot, \cdot)\|_{L^{2}\left(\mathbf{Z}^{d} \times \mathbf{R}\right)}$. Corresponding to the perturbation expansion (5.14) the function $v$ can be written as a sum

$$
\begin{aligned}
& v=\sum_{n=0}^{\infty} v_{n} \\
& \quad \text { where }\left\|v_{n}(\cdot, \cdot)\right\|_{L^{2}\left(\mathbf{Z}^{d} \times \mathbf{R}\right)} \leq \frac{1}{m^{2}}(1-\lambda / \Lambda)^{n}\|g(\cdot, \cdot)\|_{L^{2}\left(\mathbf{Z}^{d} \times \mathbf{R}\right)} .
\end{aligned}
$$


Proof. It follows from (5.16) that $v$ satisfies

$$
\left[\frac{\partial}{\partial s}-\frac{m^{2}}{2}\right] v(y, s)=\frac{1}{2} \nabla^{*} V^{\prime \prime}(\nabla \phi(y, s)) \nabla v(y, s)-g(y, s) .
$$

Multiplying (5.21) by $\overline{v(y, s)}$, summing over $y \in \mathbf{Z}^{d}$ and integrating with respect to $s$ in the interval $-T \leq s \leq T$ we see that

$$
\begin{aligned}
& \frac{m^{2}}{2} \sum_{y \in \mathbf{Z}^{d}} \int_{-T}^{T} d s|v(y, s)|^{2} \\
& \quad \leq \frac{1}{2} \sum_{y \in \mathbf{Z}^{d}}\left\{|v(y, T)|^{2}-|v(y,-T)|^{2}\right\}+\Re\left[\sum_{y \in \mathbf{Z}^{d}} \int_{-T}^{T} d s \overline{v(y, s)} g(y, s)\right] .
\end{aligned}
$$

Now from (5.19) we have that for $s \in \mathbf{R}$,

$$
\begin{aligned}
\|v(\cdot, s)\|_{L^{2}\left(\mathbf{Z}^{d}\right)} & \leq \int_{0}^{\infty} e^{-m^{2} t / 2}\|g(\cdot, s+t)\|_{L^{2}\left(\mathbf{Z}^{d}\right)} d t \\
& \leq \frac{1}{m}\left[\int_{0}^{\infty} d t\|g(\cdot, s+t)\|_{L^{2}\left(\mathbf{Z}^{d}\right)}^{2}\right]^{1 / 2} .
\end{aligned}
$$

Since $g \in L^{2}\left(\mathbf{Z}^{d} \times \mathbf{R}\right)$ it follows that the last expression on the RHS of (5.23) vanishes as $s \rightarrow \infty$, whence $\lim _{T \rightarrow \infty}\|v(\cdot, T)\|_{L^{2}\left(\mathbf{Z}^{d}\right)}=0$. We similarly conclude that $\lim _{T \rightarrow \infty}\|v(\cdot,-T)\|_{L^{2}\left(\mathbf{Z}^{d}\right)}=0$. Letting $T \rightarrow \infty$ in (5.22) and using the Schwarz inequality we see that if $g \in L^{2}\left(\mathbf{Z}^{d} \times \mathbf{R}\right)$ then $v$ is also in $L^{2}\left(\mathbf{Z}^{d} \times \mathbf{R}\right)$ and their norms are related as stated.

To prove (5.20), we observe that

$$
\left[\frac{\partial}{\partial s}-\frac{m^{2}}{2}\right] v_{0}(y, s)=\frac{\Lambda}{2} \nabla^{*} \nabla v_{0}(y, s)-g(y, s),
$$

and that for $n \geq 1$,

$$
\left[\frac{\partial}{\partial s}-\frac{m^{2}}{2}\right] v_{n}(y, s)=\frac{\Lambda}{2}\left[\nabla^{*} \nabla v_{n}(y, s)-\nabla^{*} \tilde{\mathbf{b}}_{V}(\nabla \phi(y, s)) \nabla v_{n-1}(y, s)\right] .
$$

Arguing as in the previous paragraph, we see from (5.24) that

$$
\begin{aligned}
& \frac{m^{2}}{4}\left\|v_{0}(\cdot, \cdot)\right\|_{L^{2}\left(\mathbf{Z}^{d} \times \mathbf{R}\right)}^{2}+\frac{\Lambda}{2}\left\|\nabla v_{0}(\cdot, \cdot)\right\|_{L^{2}\left(\mathbf{Z}^{d} \times \mathbf{R}, \mathbf{C}^{d}\right)}^{2} \\
& \quad \leq \frac{1}{m^{2}}\|g(\cdot, \cdot)\|_{L^{2}\left(\mathbf{Z}^{d} \times \mathbf{R}\right)}^{2} \cdot
\end{aligned}
$$

Similarly we have from (5.25) that for $n \geq 1$,

$$
\begin{aligned}
& \frac{m^{2}}{2}\left\|v_{n}(\cdot, \cdot)\right\|_{L^{2}\left(\mathbf{Z}^{d} \times \mathbf{R}\right)}^{2}+\frac{\Lambda}{4}\left\|\nabla v_{n}(\cdot, \cdot)\right\|_{L^{2}\left(\mathbf{Z}^{d} \times \mathbf{R}, \mathbf{C}^{d}\right)}^{2} \\
& \quad \leq \frac{\Lambda}{4}(1-\lambda / \Lambda)^{2}\left\|\nabla v_{n-1}(\cdot, \cdot)\right\|_{L^{2}\left(\mathbf{Z}^{d} \times \mathbf{R}, \mathbf{C}^{d}\right)}^{2}
\end{aligned}
$$

The inequality in (5.20) easily follows from (5.26), (5.27). 
REMARK 3. The result of Lemma 5.1 holds if $\mathbf{Z}^{d}$ is replaced by a periodic cube $Q$ with the Green's function $G$ replaced by the periodic Green's function $G_{Q}$ of (5.7) with perturbation expansion (5.15).

In [12] - see equation (6.31) - we considered vector valued functions $F(\phi)$ of fields $\phi: \mathbf{Z}^{d} \times \mathbf{R} \rightarrow \mathbf{R}$ and defined the field derivative of $F$ at $\phi$ to be the function $d F(\cdot, \cdot ; \phi)$ with domain $\mathbf{Z}^{d} \times \mathbf{R}$ which satisfies

$$
\lim _{\varepsilon \rightarrow 0}[F(\phi+\varepsilon h)-F(\phi)] / \varepsilon=\sum_{y \in \mathbf{Z}^{d}} \int_{-\infty}^{\infty} d s d F(y, s ; \phi) h(y, s)
$$

for all continuous functions $h: \mathbf{Z}^{d} \times \mathbf{R} \rightarrow \mathbf{R}$ with compact support. Let $\tilde{\mathbf{b}}$ : $\mathbf{R} \rightarrow \mathbf{R}^{d(d+1) / 2}$ be a $C^{1}$ function taking values in the symmetric $d \times d$ matrices such that $\|\tilde{\mathbf{b}}\|_{\infty}+\|D \tilde{\mathbf{b}}\|_{\infty}<\infty$ and define $\mathbf{b}(\cdot)$ in (4.13) as a function of fields $\phi: \mathbf{Z}^{d} \times \mathbf{R} \rightarrow \mathbf{R}$ by setting $\mathbf{b}(\phi)=\tilde{\mathbf{b}}(\phi(0,0))$. It follows from (4.11), (4.13) that for $v \in \mathbf{C}^{d}$ one has $T_{k, \Im \xi, \Re \eta} g(\Re \xi, \Im \eta, \phi) v=F(\phi)$ where

$$
\begin{aligned}
F(\phi)= & \Lambda^{k-1}\left\{\prod_{j=0}^{k-1} \sum_{x_{j} \in \mathbf{Z}^{d}} \int_{-\infty}^{\infty} d t_{j}\right\} \\
& \times \exp \left[-\eta\left(t_{k-1}-t_{0}\right)-i\left(x_{k-1}-x_{0}\right) \cdot \xi\right] \\
& \times g\left(x_{0}, t_{0}\right) \mathcal{P} \tilde{\mathbf{b}}\left(\phi\left(x_{0},-t_{0}\right)\right) \\
& \times \prod_{j=1}^{k-1}\left\{\nabla \nabla^{*} G\left(x_{j}-x_{j-1}, \Lambda\left(t_{j}-t_{j-1}\right)\right)\right\}^{*} \mathcal{P} \tilde{\mathbf{b}}\left(\phi\left(x_{j},-t_{j}\right)\right) v
\end{aligned}
$$

In (5.29), we have extended the domain of the Green's function $G(x, t)$ defined by (4.9) for $t \geq 0$ to $t<0$ by setting $G(x, t)=0, t<0$. The operator $I-\mathcal{P}$ is now any linear operator taking $d \times d$ symmetric matrix valued functions $\mathbf{b}(\cdot)$ of $\phi: \mathbf{Z}^{d} \times \mathbf{R} \rightarrow \mathbf{R}$ to a constant matrix which has the property that $\|(I-\mathcal{P}) \mathbf{b}\|_{\infty} \leq \sup _{\phi: \mathbf{Z}^{d} \times \mathbf{R} \rightarrow \mathbf{R}}\|\mathbf{b}(\phi)\|_{\infty}$. We can see from (4.10) that there exists $C_{1}>0$ depending only on $d$ such that if $(\xi, \eta)$ satisfies the inequality

$$
0<\Re \eta<\Lambda, \quad|\Im \xi|<C_{1} \sqrt{\Re \eta / \Lambda},
$$

and $g \in L^{1}\left(\mathbf{Z}^{d} \times \mathbf{R}, \mathbf{C}^{d} \otimes \mathbf{C}^{d}\right)$ then $F(\phi) \in \mathbf{C}^{d}$ is bounded by

$$
|F(\phi)| \leq C \Lambda^{k-1}\|\tilde{\mathbf{b}}\|_{\infty}^{k}[\Re \eta]^{-(k-1)}\|g\|_{1}|v|,
$$

where the constant $C$ depends only on $d, k$. Furthermore, $F$ is differentiable in the sense of (5.28) and the field derivative is given by the formula

$$
\begin{aligned}
& d F(y, s ; \phi) \\
& \quad=\Lambda^{k-1}\left\{\prod_{j=0}^{k-1} \sum_{x_{j} \in \mathbf{Z}^{d}} \int_{-\infty}^{\infty} d t_{j}\right\} \exp \left[-\eta\left(t_{k-1}-t_{0}\right)-i\left(x_{k-1}-x_{0}\right) \cdot \xi\right]
\end{aligned}
$$




$$
\begin{aligned}
& \times\left[g\left(x_{0}, t_{0}\right) \delta\left(x_{0}-y, t_{0}+s\right) D \tilde{\mathbf{b}}\left(\phi\left(x_{0},-t_{0}\right)\right)\right. \\
& \times \prod_{j=1}^{k-1}\left\{\nabla \nabla^{*} G\left(x_{j}-x_{j-1}, \Lambda\left(t_{j}-t_{j-1}\right)\right)\right\}^{*} \mathcal{P} \tilde{\mathbf{b}}\left(\phi\left(x_{j},-t_{j}\right)\right) v+\cdots \\
& +g\left(x_{0}, t_{0}\right) \tilde{\mathbf{b}}\left(\phi\left(x_{0},-t_{0}\right)\right) \prod_{j=1}^{k-2}\left\{\nabla \nabla^{*} G\left(x_{j}-x_{j-1}, \Lambda\left(t_{j}-t_{j-1}\right)\right)\right\}^{*} \\
& \times \tilde{\mathbf{b}}\left(\phi\left(x_{j},-t_{j}\right)\right) \delta\left(x_{k-1}-y, t_{k-1}+s\right) \\
& \times\left\{\nabla \nabla^{*} G\left(x_{k-1}-x_{k-2}, \Lambda\left(t_{k-1}-t_{k-2}\right)\right)\right\}^{*} \\
& \left.\times D \tilde{\mathbf{b}}\left(\phi\left(x_{k-1},-t_{k-1}\right)\right) v\right]
\end{aligned}
$$

for $(y, s) \in \mathbf{Z}^{d} \times \mathbf{R}$, where $\delta(x, t)=\delta(x) \delta(t)$ is the product of the Kronecker and Dirac delta functions. If $g$ is also in $L^{2}\left(\mathbf{Z}^{d} \times \mathbf{R}, \mathbf{C}^{d} \otimes \mathbf{C}^{d}\right)$ then $d F(\cdot, \cdot ; \phi) \in$ $L^{2}\left(\mathbf{Z}^{d} \times \mathbf{R}, \mathbf{C}^{d}\right)$ and from $(4.10)$ we see that

$$
\begin{aligned}
& \|d F(\cdot, \cdot ; \phi)\|_{2} \\
& \leq C \Lambda^{k-1}\|D \tilde{\mathbf{b}}\|_{\infty}\|\tilde{\mathbf{b}}\|_{\infty}^{k-1}[\Re \eta]^{-(k-1)} \\
& \quad \times\left[\|g\|_{2}+\Re \eta\|g\|_{1}\left\{\sum_{x \in \mathbf{Z}^{d}} \int_{0}^{\infty} d t\left|\nabla \nabla^{*} G(x, \Lambda t)\right|^{2}\right\}^{1 / 2}\right]|v|,
\end{aligned}
$$

where $C$ depends only on $d, k$.

LEMMA 5.2. Let $\left(\Omega, \mathcal{F}, P_{m}\right)$ with $m>0$ be the environment of massive fields $\phi: \mathbf{Z}^{d} \times \mathbf{R} \rightarrow \mathbf{R}$ defined by (2.12), (1.1) and $g: \mathbf{Z}^{d} \times \mathbf{R} \rightarrow \mathbf{C}^{d} \otimes \mathbf{C}^{d}$ a continuous function of compact support. Then there exists $C_{1}>0$ depending only on $d$ such that if $(\xi, \eta)$ lies in the region (5.30) the operator of (4.13) satisfies the inequality

$$
\begin{aligned}
& \left\langle\left|T_{k, \Im \xi, \Re \eta} g(\Re \xi, \Im \eta, \cdot) v\right|^{2}\right\rangle \\
& \quad \leq\left\langle\sum_{y \in \mathbf{Z}^{d}} \int_{-\infty}^{\infty} d s\left|\sum_{x \in \mathbf{Z}^{d}} \int_{s}^{\infty} d t e^{-m^{2}(t-s) / 2} G(y, s ; x, t, \phi) d F(x, t ; \phi)\right|^{2}\right\rangle
\end{aligned}
$$

where $G$ is the Green's function defined by (5.4), and $d F$ is the field derivative (5.32).

Proof. It follows from (5.31) that the LHS of (5.34) is the expectation of a bounded function. From Lemma 5.1 and (5.33), we see that the RHS is also the expectation of a bounded function. To prove (5.34), we use the Poincaré inequality (5.2) and the formula (5.8) for the Malliavin derivative. Thus, let $Q \subset \mathbf{Z}^{d}$ be the periodic cube with side of length $L$ and $\phi(x, t), x \in Q, t \geq 0$, the solution to the initial value problem (5.1). For $T>0$, we denote by $\phi_{T}$ 
the periodic field $\phi_{T}: Q \times \mathbf{R} \rightarrow \mathbf{R}$ defined by $\phi_{T}(x, t)=\phi(x, T+t), x \in Q$, $t \geq-T$ where $\phi$ is the solution to (5.1), and $\phi_{T}(x, t)=0, x \in Q, t<-T$. We extend the field $\phi_{T}$ to a periodic field $\phi_{T}: \mathbf{Z}^{d} \times \mathbf{R} \rightarrow \mathbf{R}$. From (5.8), we have that for the function $F$ of (5.29) if $y \in Q$ and $s>-T$ then

$$
\begin{aligned}
& D_{\mathrm{Mal}} F\left(y, T+s ; \phi_{T}\right) \\
& \quad=\sum_{x \in Q} \int_{s}^{\infty} d t e^{-m^{2}(t-s) / 2} G_{Q}\left(y, s ; x, t, \phi_{T}\right) d F_{Q}\left(x, t ; \phi_{T}\right),
\end{aligned}
$$

where $d F_{Q}$ is given in terms of (5.32) by

$$
d F_{Q}(x, t ; \phi)=\sum_{n \in \mathbf{Z}^{d}} d F(x+L n, t ; \phi) .
$$

It is easy to see from $(5.32)$ that $\left\|d F_{Q}(\cdot, \cdot ; \phi)\right\|_{\infty} \leq C$ for some constant independent of $\phi$ so the RHS of (5.35) is bounded.

The invariant measure associated with the Markov process defined by the $\mathrm{SDE}$ (5.1) is given by the formula

$$
\exp \left[-\sum_{x \in Q} V(\nabla \phi(x))+m^{2} \phi(x)^{2} / 2\right] \prod_{x \in Q} d \phi(x) / \text { normalization. }
$$

We denote the probability space for the corresponding stationary process $\phi(x, t), x \in Q, t \in \mathbf{R}$ by $\left(\Omega_{Q}, \mathcal{F}_{Q}, P_{Q, m}\right)$ and expectation with respect to the measure $P_{Q, m}$ by $\langle\cdot\rangle_{\Omega_{Q, m}}$. Evidently $\left(\Omega_{Q}, \mathcal{F}_{Q}, P_{Q, m}\right)$ is invariant with respect to the translation operators $\tau_{x, t}: \Omega_{Q} \rightarrow \Omega_{Q}, x \in \mathbf{Z}^{d}, t \in \mathbf{R}$. Our first goal will be to obtain a version of the inequality (5.34) for the operator $T_{k, \Im \xi, \Re \eta}$ of (4.13) when the random environment is given by $\left(\Omega_{Q}, \mathcal{F}_{Q}, P_{Q, m}\right)$. To do this, we use the fact (see Appendix A for a proof) that for any $N \geq 1$, continuous bounded function $f: \mathbf{R}^{N} \rightarrow \mathbf{C}$, and $\left(x_{1}, t_{1}\right), \ldots,\left(x_{N}, t_{N}\right) \in Q \times \mathbf{R}$,

$$
\begin{aligned}
\lim _{T \rightarrow \infty} & \left\langle f\left(\phi_{T}\left(x_{1}, t_{1}\right), \ldots, \phi_{T}\left(x_{N}, t_{N}\right)\right)\right\rangle_{\Omega_{Q, \text { Mal }}} \\
& =\left\langle f\left(\phi\left(x_{1}, t_{1}\right), \ldots, \phi\left(x_{N}, t_{N}\right)\right)\right\rangle_{\Omega_{Q, m}} .
\end{aligned}
$$

It follows from (5.38) that for any $d \times d$ constant matrices $A_{1}, \ldots, A_{N+1}$,

$$
\begin{gathered}
\lim _{T \rightarrow \infty}\left\langle v^{*} \prod_{j=1}^{N} A_{j} \mathcal{P} \tilde{\mathbf{b}}\left(\phi_{T}\left(x_{j},-t_{j}\right)\right) A_{N+1} v\right\rangle_{\Omega_{Q, \text { Mal }}} \\
=\left\langle v^{*} \prod_{j=1}^{N} A_{j} \mathcal{P} \tilde{\mathbf{b}}\left(\phi\left(x_{j},-t_{j}\right)\right) A_{N+1} v\right\rangle_{\Omega_{Q, m}},
\end{gathered}
$$

where $I-\mathcal{P}$ on the LHS of (5.39) denotes expectation with respect to $\langle\cdot\rangle_{\Omega_{Q, \mathrm{Mal}}}$ and on the RHS expectation with respect to $\langle\cdot\rangle_{\Omega_{Q, m}}$. We conclude from (5.39), 
Fubini's theorem and the dominated convergence theorem that

$$
\begin{aligned}
\lim _{T \rightarrow \infty}\left\langle\left|F\left(\phi_{T}\right)\right|^{2}\right\rangle_{\Omega_{Q, \mathrm{Mal}}} & =\left\langle|F(\phi)|^{2}\right\rangle_{\Omega_{Q, m}} \\
& =\left\langle\left|T_{k, \Im \xi, \Re \eta} g(\Re \xi, \Im \eta, \cdot) v\right|^{2}\right\rangle_{\Omega_{Q, m}} .
\end{aligned}
$$

Next, we see from the Poincaré inequality (5.2) and (5.35) that

$$
\begin{aligned}
& \left\langle\left|F\left(\phi_{T}\right)\right|^{2}\right\rangle_{\Omega_{Q, \text { Mal }}} \\
& \leq\left\langle\left\langle\sum_{y \in Q} \int_{-T}^{\infty} d s\right| \sum_{x \in Q} \int_{s}^{\infty} d t e^{-m^{2}(t-s) / 2}\right. \\
& \left.\quad \times\left. G_{Q}\left(y, s ; x, t, \phi_{T}\right) d F_{Q}\left(x, t ; \phi_{T}\right)\right|^{2}\right\rangle_{\Omega_{Q, \mathrm{Mal}}} .
\end{aligned}
$$

We assume that $L$ is sufficiently large so that the support of $g$ is contained in $Q \times \mathbf{R}$. It is easy to see then that $d F_{Q}(y, s ; \phi)$ is given by the RHS of (5.32) with $\mathbf{Z}^{d}$ replaced by $Q$ and the function $G(x, \Lambda t)$ replaced by the corresponding periodic Green's function on $Q$. Hence, $\left\|d F_{Q}(\cdot, \cdot, \phi)\right\|_{L^{2}\left(Q \times \mathbf{R}, \mathbf{C}^{d}\right)}$ is bounded by the periodic version of the RHS of (5.33). From this, we see that $\limsup _{Q \rightarrow \mathbf{Z}^{d}}\left\|d F_{Q}(\cdot, \cdot, \phi)\right\|_{L^{2}\left(Q \times \mathbf{R}, \mathbf{C}^{d}\right)}<\infty$. Observe now that we can argue as in the proof of (5.40) to conclude that if $G_{n, Q}, n=0,1,2, \ldots$, denote the terms in the perturbation expansion (5.15) and $N \geq 0$ then

$$
\begin{aligned}
\lim _{T \rightarrow \infty} & \left\langle\sum_{y \in Q} \int_{-T}^{\infty} d s\right| \sum_{x \in Q} \int_{s}^{\infty} d t e^{-m^{2}(t-s) / 2} \\
& \left.\times\left.\sum_{n=0}^{N} G_{n, Q}\left(y, s ; x, t, \phi_{T}\right) d F_{Q}\left(x, t ; \phi_{T}\right)\right|^{2}\right\rangle_{\Omega_{Q, \text { Mal }}} \\
= & \left\langle\sum_{y \in Q} \int_{-\infty}^{\infty} d s\right| \sum_{x \in Q} \int_{s}^{\infty} d t e^{-m^{2}(t-s) / 2} \\
& \left.\times\left.\sum_{n=0}^{N} G_{n, Q}(y, s ; x, t, \phi) d F_{Q}(x, t ; \phi)\right|^{2}\right\rangle_{\Omega_{Q, m}} .
\end{aligned}
$$

It follows from (5.40), (5.42) and the periodic version of Lemma 5.1 that

$$
\begin{aligned}
& \left\langle\left|T_{k, \Im \xi, \Re \eta} g(\Re \xi, \Im \eta, \cdot) v\right|^{2}\right\rangle_{\Omega_{Q, m}} \\
& \leq\left\langle\sum_{y \in Q} \int_{-\infty}^{\infty} d s\right| \sum_{x \in Q} \int_{s}^{\infty} d t e^{-m^{2}(t-s) / 2} \\
& \left.\quad \times\left. G_{Q}(y, s ; x, t, \phi) d F_{Q}(x, t ; \phi)\right|^{2}\right\rangle_{\Omega_{Q, m}} .
\end{aligned}
$$


Finally, we let $Q \rightarrow \mathbf{Z}^{d}$ in (5.43) to obtain (5.34). We denote by $\langle\cdot\rangle_{\Omega_{m}}$ expectation with respect to the stationary process defined by (2.12), (1.1). It was proved in [20]) (see also [10]) that for any $N \geq 1$, continuous bounded function $f: \mathbf{R}^{N} \rightarrow \mathbf{C}$, and $\left(x_{1}, t_{1}\right), \ldots,\left(x_{N}, t_{N}\right) \in Q \times \mathbf{R}$,

$$
\begin{aligned}
& \lim _{Q \rightarrow \mathbf{Z}^{d}}\left\langle f\left(\phi\left(x_{1}, t_{1}\right), \ldots, \phi\left(x_{N}, t_{N}\right)\right)\right\rangle_{\Omega_{Q, m}} \\
& \quad=\left\langle f\left(\phi\left(x_{1}, t_{1}\right), \ldots, \phi\left(x_{N}, t_{N}\right)\right)\right\rangle_{\Omega_{m}} .
\end{aligned}
$$

Hence, we can using (5.44) argue as with the $T \rightarrow \infty$ limit to conclude that (5.34) holds for any $m>0$.

For $\xi \in \mathbf{R}^{d}$ and $u: \mathbf{Z}^{d} \rightarrow \mathbf{C}$ we denote by $\nabla_{\xi} u: \mathbf{Z}^{d} \rightarrow \mathbf{C}^{d}$ the column vector $\nabla_{\xi} u(z)=\left[\nabla_{1, \xi} u(z), \ldots, \nabla_{j, \xi} u(z)\right], z \in \mathbf{Z}^{d}$, where $\nabla_{j, \xi} u(z)=e^{-i \mathbf{e}_{j} \cdot \xi} u\left(z+\mathbf{e}_{j}\right)-$ $u(z), z \in \mathbf{Z}^{d}, j=1, \ldots, d$. The column operator $\nabla_{\xi}$ has adjoint $\nabla_{\xi}^{*}$ which is a row operator. If $f \in L^{2}\left(\mathbf{Z}^{d} \times \mathbf{R}, \mathbf{C}^{d}\right)$ and $\xi \in \mathbf{R}^{d}, \eta \in \mathbf{C}$ with $\Re \eta>0$ there is a unique solution $u(\xi, \eta, \cdot) \in L^{2}\left(\mathbf{Z}^{d} \times \mathbf{R}\right)$ to the $\mathrm{PDE}$

$$
\begin{aligned}
& \frac{1}{\Lambda}\left[\eta-\frac{\partial}{\partial r}\right] u(\xi, \eta, z, r)+\nabla_{\xi}^{*} \nabla_{\xi} u(\xi, \eta, z, r)=\nabla_{\xi}^{*} f(z, r), \\
& \quad z \in \mathbf{Z}^{d}, r \in \mathbf{R} .
\end{aligned}
$$

Furthermore, we have that

$$
\begin{aligned}
& \frac{\Re \eta}{\Lambda}\|u(\xi, \eta, \cdot)\|_{L^{2}\left(\mathbf{Z}^{d} \times \mathbf{R}\right)}^{2}+\left\|\nabla_{\xi} u(\xi, \eta, \cdot)\right\|_{L^{2}\left(\mathbf{Z}^{d} \times \mathbf{R}, \mathbf{C}^{d}\right)}^{2} \\
& \quad \leq\|f(\cdot)\|_{L^{2}\left(\mathbf{Z}^{d} \times \mathbf{R}, \mathbf{C}^{d}\right)}^{2} .
\end{aligned}
$$

We also see similarly to (4.11) that $\nabla_{\xi} u(\xi, \eta, \cdot)=\tilde{T}_{\xi, \eta} f(\cdot)$ where (5.47) $\tilde{T}_{\xi, \eta} f(z, r)$

$$
\begin{aligned}
= & \Lambda \int_{0}^{\infty} e^{-\eta s} d s \sum_{y \in \mathbf{Z}^{d}}\left\{\nabla \nabla^{*} G(y, \Lambda s)\right\}^{*} \exp [-i y . \xi] f(z+y, r+s), \\
& z \in \mathbf{Z}^{d}, r \in \mathbf{R} .
\end{aligned}
$$

From Lemma 2.1 of [12], we have that $u$ regarded as a function $(\xi, \eta) \rightarrow$ $L^{2}\left(\mathbf{Z}^{d} \times \mathbf{R}\right)$ has an analytic continuation to the region (5.30) with $C_{1}>0$ depending only on $d$. For $(\xi, \eta)$ in this region there is a constant $C_{2}$ depending only on $d$ such that

$$
\begin{aligned}
& \frac{\Re \eta}{2 \Lambda}\|u(\xi, \eta, \cdot)\|_{L^{2}\left(\mathbf{Z}^{d} \times \mathbf{R}\right)}^{2}+\left\|\nabla_{\Re \xi} u(\xi, \eta, \cdot)\right\|_{L^{2}\left(\mathbf{Z}^{d} \times \mathbf{R}, \mathbf{C}^{d}\right)}^{2} \\
& \quad \leq\left[1+C_{2}|\Im \xi|^{2} /(\Re \eta / \Lambda)\right]\|f(\cdot)\|_{L^{2}\left(\mathbf{Z}^{d} \times \mathbf{R}, \mathbf{C}^{d}\right)}^{2} .
\end{aligned}
$$

For $\phi: \mathbf{Z}^{d} \times \mathbf{R} \rightarrow \mathbf{R}$ a continuous function, we extend the corresponding Green's function $G(y, s ; x, t, \phi)$ defined by (5.4) for $s \leq t$ to $s>t$ by setting 
$G(y, s ; x, t, \phi)=0$ when $s>t$. For $\xi \in \mathbf{R}^{d}, \eta \in \mathbf{C}, v \in \mathbf{C}^{d}$, with $\Re \eta>0$ let $f_{2}(\xi, \eta, z, r, \phi), z \in \mathbf{Z}^{d}, r \in \mathbf{R}$ with range in $\mathbf{C}^{d}$ be defined by

$$
f_{2}(\xi, \eta, z, r, \phi)=D \tilde{\mathbf{b}}(\phi(z,-r)) v e^{m^{2} r / 2} G(0,0 ; z,-r, \phi) .
$$

It is evident from (5.3), (5.4) that $f_{2}(\xi, \eta, \cdot, \cdot, \phi)$ is in $L^{2}\left(\mathbf{Z}^{d} \times \mathbf{R}\right)$, whence we can define the function $u_{2}(\xi, \eta, z, r, \phi)$ as the solution to $(5.45)$ with $f(\cdot, \cdot)=$ $f_{2}(\xi, \eta, \cdot, \cdot \phi)$. Let $\mathbf{b}(\phi)=\tilde{\mathbf{b}}(\phi(0,0))$ and for $k=2,3, \ldots$, set

$$
\partial_{\xi} F_{k}(\xi, \eta, \phi)=\left[\mathcal{P} T_{\xi, \eta} \mathbf{b}(\cdot)\right]^{k-1} v
$$

where $T_{\xi, \eta}$ is the operator (4.11). We then inductively define functions $f_{k}, u_{k}$ for $k=3,4, \ldots$, by the formula

$$
\begin{aligned}
& f_{k}(\xi, \eta, z, r, \phi) \\
& =D \tilde{\mathbf{b}}(\phi(z,-r)) \partial_{\xi} F_{k-1}\left(\xi, \eta, \tau_{z,-r} \phi\right) e^{m^{2} r / 2} G(0,0 ; z,-r, \phi) \\
& \quad+\tilde{\mathbf{b}}(\phi(z,-r)) \nabla_{\xi} u_{k-1}(\xi, \eta, z, r, \phi),
\end{aligned}
$$

where for $k=3,4, \ldots$, the function $u_{k}(\xi, \eta, z, r, \phi)$ is the solution to (5.45) with $f(\cdot, \cdot)=f_{k}(\xi, \eta, \cdot, \cdot, \phi)$. The $u_{k}(\xi, \eta, z, r, \phi)$ and $f_{k}(\xi, \eta, z, r, \phi)$ for $\xi \in \mathbf{C}^{d}$ are defined by analytic continuation from their values when $\xi \in \mathbf{R}^{d}$.

LEMMA 5.3. Let $G$ be the Green's function defined by (5.4) and for any $k \geq$ $2, v \in \mathbf{C}^{d}$, let $d F$ be the function (5.32). Then there exists $C_{1}>0$ depending only on $d$ such that for $(\xi, \eta)$ in the region (5.30) the following identity holds:

$$
\begin{aligned}
& \sum_{y \in \mathbf{Z}^{d}} \int_{-r}^{\infty} d s e^{-m^{2}(s+r) / 2} G\left(z,-r ; y, s, \tau_{-z, r} \phi\right) d F\left(y, s ; \tau_{-z, r} \phi\right) \\
& =\sum_{x \in \mathbf{Z}^{d}} \int_{-\infty}^{\infty} d t g(x, t) e^{m^{2}(t-r) / 2} \\
& \quad \times G(0,0 ; x-z, r-t, \phi) D \tilde{\mathbf{b}}(\phi(x-z, r-t)) \partial_{\xi} F_{k}\left(\xi, \eta, \tau_{x-z, r-t} \phi\right) \\
& \quad+\sum_{x \in \mathbf{Z}^{d}} \int_{-\infty}^{\infty} d t g(x, t) \tilde{\mathbf{b}}(\phi(x-z, r-t)) \nabla_{\xi} u_{k}(\xi, \eta, x-z, t-r, \phi) .
\end{aligned}
$$

Proof. We note that the first term on the RHS of (5.52) comes from the sum which contains $\delta\left(x_{0}-y, t_{0}+s\right)$ on the RHS of $(5.32)$, in which we make the change of variables $(y, s) \leftrightarrow(x,-t)$. The remaining part of the RHS of (5.32) is the same as

$$
\begin{aligned}
& \sum_{x \in \mathbf{Z}^{d}} \int_{-\infty}^{\infty} d t g(x, t) \tilde{\mathbf{b}}(\phi(x-z, r-t)) \sum_{y \in \mathbf{Z}^{d}} \int_{-r}^{\infty} d s e^{-m^{2}(s+r) / 2} \\
& \quad \times G\left(z,-r ; y, s, \tau_{-z, r} \phi\right) d H_{k}\left(y-x, s+t ; \tau_{x-z, r-t} \phi\right),
\end{aligned}
$$

where $d H_{k}$ is the field derivative of the function

$$
H_{k}(\phi)=\left[\mathcal{P} T_{\xi, \eta} \mathbf{b}(\cdot)\right]^{k-1} v .
$$


Observe now that

$$
\begin{aligned}
\sum_{y \in \mathbf{Z}^{d}} & \int_{-r}^{\infty} d s e^{-m^{2}(s+r) / 2} \\
& \times G\left(z,-r ; y, s, \tau_{-z, r} \phi\right) d H_{k}\left(y-x, s+t ; \tau_{x-z, r-t} \phi\right) \\
= & \sum_{y \in \mathbf{Z}^{d}} \int_{0}^{\infty} d s e^{-m^{2} s / 2} \\
& \times G(0,0 ; y, s, \phi) d H_{k}\left(y-(x-z), s+(t-r) ; \tau_{x-z, r-t} \phi\right),
\end{aligned}
$$

so the expression is just a function of $(x-z, t-r, \phi)$. We show by induction that for $k \geq 2$,

$$
\begin{aligned}
& \nabla_{\xi} u_{k}(\xi, \eta, x, t, \phi) \\
& \quad=\sum_{y \in \mathbf{Z}^{d}} \int_{0}^{\infty} d s e^{-m^{2} s / 2} G(0,0 ; y, s, \phi) d H_{k}\left(y-x, s+t ; \tau_{x,-t} \phi\right) .
\end{aligned}
$$

Hence the identity (5.52) follows from (5.55).

To prove $(5.56)$, we first show that it holds for $k=2$. To see this, we note from (4.11) that $d H_{2}$ is given by the formula

$$
d H_{2}(y, s ; \phi)=\Lambda\left\{\nabla \nabla^{*} G(y,-\Lambda s)\right\}^{*} \exp [\eta s-i y . \xi] D \tilde{\mathbf{b}}(\phi(y, s)) v .
$$

Now (5.56) for $k=2$ follows from (5.47), (5.49), (5.57). To do the induction step, we use the identity

$$
\begin{aligned}
d H_{k+1} & (y, s ; \phi) \\
= & \Lambda\left\{\nabla \nabla^{*} G(y,-\Lambda s)\right\}^{*} \exp [\eta s-i y \cdot \xi] D \tilde{\mathbf{b}}(\phi(y, s)) \partial_{\xi} F_{k}\left(\xi, \eta, \tau_{y, s} \phi\right) \\
& +\Lambda \int_{0}^{\infty} e^{-\eta r} d r \sum_{z \in \mathbf{Z}^{d}}\left\{\nabla \nabla^{*} G(z, \Lambda r)\right\}^{*} \\
& \times \exp [-i z \cdot \xi] \tilde{\mathbf{b}}(\phi(z,-r)) d H_{k}\left(y-z, s+r ; \tau_{z,-r} \phi\right) .
\end{aligned}
$$

From (5.58) and the induction hypothesis (5.56), we conclude that

$$
\begin{aligned}
& \sum_{y \in \mathbf{Z}^{d}} \int_{0}^{\infty} d s e^{-m^{2} s / 2} G(0,0 ; y, s, \phi) d H_{k+1}\left(y-x, s+t ; \tau_{x,-t} \phi\right) \\
& =\tilde{T}_{\xi, \eta}\left[f_{k+1}(\xi, \eta, \cdot, \cdot, \phi)\right](x, t)=\nabla_{\xi} u_{k+1}(\xi, \eta, x, t, \phi) .
\end{aligned}
$$

In order to prove Lemma 4.1 we shall need to use a parabolic version of Meyer's theorem [32].

LEMmA 5.4. Let $\mathcal{H}$ be a Hilbert space and for $1<p<\infty$ let $L^{p}\left(\mathbf{Z}^{d} \times \mathbf{R}, \mathcal{H}\right)$ be the space of $p$ integrable functions $f: \mathbf{Z}^{d} \times \mathbf{R} \rightarrow \mathcal{H}$ where the $L^{p}$ norm of $f$ 
is defined by

$$
\|f\|_{p}^{p}=\sum_{y \in \mathbf{Z}^{d}} \int_{-\infty}^{\infty} d s\|f(y, s)\|_{\mathcal{H}}^{p} .
$$

Then there exists a constant $C_{1}>0$ depending only on $d$ such that if $(\xi, \eta)$ lies in the region (5.30) the operator $\tilde{T}_{\xi, \eta}$ of $(5.47)$ is bounded on $L^{p}\left(\mathbf{Z}^{d} \times \mathbf{R}, \mathcal{H}\right)$ for $1<p<\infty$. Furthermore there is a constant $C_{2}>0$ depending only on $d$ such that the norm of $\tilde{T}_{\xi, \eta}$ acting on $L^{p}\left(\mathbf{Z}^{d} \times \mathbf{R}, \mathcal{H}\right)$ satisfies the inequality $\left\|\tilde{T}_{\xi, \eta}\right\|_{p} \leq[1+\delta(p)]\left(1+C_{2}|\Im \xi|^{2} /[\Re \eta / \Lambda]\right)$ where the function $\delta(\cdot)$ depends only on $d$ and $\lim _{p \rightarrow 2} \delta(p)=0$.

Proof. This follows from the argument of Lemma 5.2 and Corollary 5.1 of [12].

Proof of Lemma 4.1. We assume $g: \mathbf{Z}^{d} \times \mathbf{R} \rightarrow \mathbf{C}^{d} \otimes \mathbf{C}^{d}$ is continuous of compact support. Then from Lemma 5.2 and Lemma 5.3 we have that

$$
\begin{aligned}
& \left\langle\left|T_{k, \Im \xi, \Re \eta} g(\Re \xi, \Im \eta, \cdot) v\right|^{2}\right\rangle_{\Omega_{m}} \\
& \leq 2 \sum_{z \in \mathbf{Z}^{d}} \int_{-\infty}^{\infty} d r\langle| \sum_{x \in \mathbf{Z}^{d}} \int_{-\infty}^{\infty} d t g(x, t) e^{m^{2}(t-r) / 2} G(0,0 ; x-z, r-t, \phi) \\
& \left.\quad \times\left. D \tilde{\mathbf{b}}(\phi(x-z, r-t)) \partial_{\xi} F_{k}\left(\xi, \eta, \tau_{x-z, r-t} \phi\right)\right|^{2}\right\rangle_{\Omega_{m}} \\
& \quad+\langle| \sum_{x \in \mathbf{Z}^{d}} \int_{-\infty}^{\infty} d t g(x, t) \tilde{\mathbf{b}}(\phi(x-z, r-t)) \\
& \left.\quad \times\left.\mathcal{P} \nabla_{\xi} u_{k}(\xi, \eta, x-z, t-r, \phi)\right|^{2}\right\rangle_{\Omega_{m}}
\end{aligned}
$$

Let $(\Omega, \mathcal{F}, P)$ be the massless field stationary process corresponding to the limit as $m \rightarrow 0$ of the massive field stationary processes defined by (2.12), (1.1), and denote expectation with respect to this measure by $\langle\cdot\rangle_{\Omega}$. We have from [20] (see also [10]) that if $d \geq 3$ then for any $N \geq 1$, continuous bounded function $f: \mathbf{R}^{N} \rightarrow \mathbf{C}$, and $\left(x_{1}, t_{1}\right), \ldots,\left(x_{N}, t_{N}\right) \in \mathbf{Z}^{d} \times \mathbf{R}$,

(5.62) $\lim _{m \rightarrow 0}\left\langle f\left(\phi\left(x_{1}, t_{1}\right), \ldots, \phi\left(x_{N}, t_{N}\right)\right)\right\rangle_{\Omega_{m}}=\left\langle f\left(\phi\left(x_{1}, t_{1}\right), \ldots, \phi\left(x_{N}, t_{N}\right)\right)\right\rangle_{\Omega}$.

Arguing as in Lemma 5.2, we conclude that if $d \geq 3$ then

$$
\lim _{m \rightarrow 0}\left\langle\left|T_{k, \Im \xi, \Re \eta} g(\Re \xi, \Im \eta, \cdot) v\right|^{2}\right\rangle_{\Omega_{m}}=\left\langle\left|T_{k, \Im \xi, \Re \eta} g(\Re \xi, \Im \eta, \cdot) v\right|^{2}\right\rangle_{\Omega} .
$$

Hence to prove Lemma 4.1 it will be sufficient to obtain an upper bound on the RHS of (5.61) which is independent of $m$ as $m \rightarrow 0$. 
For $\xi \in \mathbf{R}^{d}, \eta \in \mathbf{C}$ with $\Re \eta>0$ the functions $F_{k}(\xi, \eta, \phi), k=2,3, \ldots, \phi \in \Omega$, defined by (5.50) satisfy the recurrence equations

$$
\begin{aligned}
& {[\eta+\partial] F_{2}(\xi, \eta, \phi)+\Lambda \partial_{\xi}^{*} \partial_{\xi} F_{2}(\xi, \eta, \phi)=\Lambda \mathcal{P} \partial_{\xi}^{*}[\tilde{\mathbf{b}}(\phi(0,0)) v],} \\
& {[\eta+\partial] F_{k}(\xi, \eta, \phi)+\Lambda \partial_{\xi}^{*} \partial_{\xi} F_{k}(\xi, \eta, \phi)} \\
& \quad=\Lambda \mathcal{P} \partial_{\xi}^{*}\left[\tilde{\mathbf{b}}(\phi(0,0)) \partial_{\xi} F_{k-1}(\xi, \eta, \phi)\right] \quad \text { if } k>2 .
\end{aligned}
$$

Then as in (4.2) we see that $F_{k}(\xi, \eta, \cdot) \in L^{2}(\Omega)$ and

$$
\frac{\Re \eta}{\Lambda}\left\|F_{k}(\xi, \eta, \cdot)\right\|_{L^{2}(\Omega)}^{2}+\left\|\partial_{\xi} F_{k}(\xi, \eta, \cdot)\right\|_{\mathcal{H}(\Omega)}^{2} \leq(1-\lambda / \Lambda)^{2(k-1)}|v|^{2} .
$$

The $F_{k}(\xi, \eta, \phi)$ for $\xi \in \mathbf{C}^{d}$ are defined by analytic continuation from the values of $F_{k}(\xi, \eta, \phi)$ when $\xi \in \mathbf{R}^{d}$. From the continuous time version of Lemma 2.1 of [12], we see that $F_{k}$ regarded as a function $(\xi, \eta) \rightarrow L^{2}(\Omega)$ has an analytic continuation to the region (5.30) where $C_{1}$ is a constant depending only on $d$. For $(\xi, \eta)$ in this region there is a constant $C_{2}$ depending only on $d$ such that

$$
\begin{aligned}
& \frac{\Re \eta}{2 \Lambda}\left\|F_{k}(\xi, \eta, \cdot)\right\|_{L^{2}(\Omega)}^{2}+\left\|\partial_{\xi} F_{k}(\xi, \eta, \cdot)\right\|_{\mathcal{H}(\Omega)}^{2} \\
& \quad \leq(1-\lambda / \Lambda)^{2(k-1)}\left[1+C_{2}|\Im \xi|^{2} /(\Re \eta / \Lambda)\right]^{2(k-1)}|v|^{2} .
\end{aligned}
$$

We take $\mathcal{H}$ to be the Hilbert space $\mathcal{H}=L^{2}\left(\Omega, \mathbf{C}^{d}\right)$ and for $k=2,3, \ldots$, let $h_{k}: \mathbf{Z}^{d} \times \mathbf{R} \rightarrow \mathcal{H}$ be the function

$$
h_{k}(z, r, \phi)=D \tilde{\mathbf{b}}(\phi(z,-r)) \partial_{\xi} F_{k}\left(\xi, \eta, \tau_{z,-r} \phi\right) e^{m^{2} r / 2} G(0,0 ; z,-r, \phi) .
$$

Then the first term on the RHS of (5.61) is the square of the norm in $L^{2}\left(\mathbf{Z}^{d} \times\right.$ $\mathbf{R}, \mathcal{H})$ of the convolution $g * h_{k}$. It follows from (2.17) and (5.66) that if $q>1+2 / d$ then

$$
\begin{aligned}
& \left\|h_{k}\right\|_{L^{q}\left(\mathbf{Z}^{d} \times \mathbf{R}, \mathcal{H}\right)} \\
& \quad \leq C_{q} \Lambda^{-1 / q}\|D \tilde{\mathbf{b}}(\cdot)\|_{\infty}(1-\lambda / \Lambda)^{(k-1)}\left[1+C_{2}|\Im \xi|^{2} /(\Re \eta / \Lambda)\right]^{(k-1)}|v|,
\end{aligned}
$$

where the constant $C_{q}$ depends only on $d, q, \lambda / \Lambda$. Taking $q<2$ we have by Young's inequality for convolutions that if for $p=2 q /(3 q-2)$ the function $g$ is in $L^{p}\left(\mathbf{Z}^{d} \times \mathbf{R}, \mathbf{C}^{d} \otimes \mathbf{C}^{d}\right)$ with norm (4.14) then $g * h_{k}$ is in $L^{2}\left(\mathbf{Z}^{d} \times \mathbf{R}, \mathcal{H}\right)$ and

$$
\left\|g * h_{k}\right\|_{L^{2}\left(\mathbf{Z}^{d} \times \mathbf{R}, \mathcal{H}\right)} \leq C\|g\|_{L^{p}\left(\mathbf{Z}^{d} \times \mathbf{R}, \mathbf{C}^{d} \otimes \mathbf{C}^{d}\right)}\left\|h_{k}\right\|_{L^{q}\left(\mathbf{Z}^{d} \times \mathbf{R}, \mathcal{H}\right)},
$$

for a constant $C$ depending only on $d$.

To bound the second term on the RHS of (5.61), we show that the function $(z, r) \rightarrow \nabla_{\xi} u_{k}(\xi, \eta, z, r, \phi)$ is in $L^{q}\left(\mathbf{Z}^{d} \times \mathbf{R}, \mathcal{H}\right)$. In fact from (2.17), (5.49) we have that

$$
\left\|f_{2}(\xi, \eta, \cdot, \cdot, \cdot)\right\|_{L^{q}\left(\mathbf{Z}^{d} \times \mathbf{R}, \mathcal{H}\right)} \leq C_{q} \Lambda^{-1 / q}\|D \tilde{\mathbf{b}}(\cdot)\|_{\infty}|v|,
$$


for a constant $C_{q}$ depending only on $q, d$. Hence, Lemma 5.4 implies that

$$
\begin{aligned}
& \left\|\nabla_{\xi} u_{2}(\xi, \eta, \cdot, \cdot, \cdot)\right\|_{L^{q}\left(\mathbf{Z}^{d} \times \mathbf{R}, \mathcal{H}\right)} \\
& \quad \leq C_{q} \Lambda^{-1 / q}\|D \tilde{\mathbf{b}}(\cdot)\|_{\infty}[1+\delta(q)]\left[1+C_{2}|\Im \xi|^{2} /(\Re \eta / \Lambda)\right]|v| .
\end{aligned}
$$

From (5.51), we similarly have that for $k \geq 3$,

$$
\begin{aligned}
& \left\|f_{k}(\xi, \eta, \cdot, \cdot, \cdot)\right\|_{L^{q}\left(\mathbf{Z}^{d} \times \mathbf{R}, \mathcal{H}\right)} \\
& \quad \leq C_{q} \Lambda^{-1 / q}\|D \tilde{\mathbf{b}}(\cdot)\|_{\infty}(1-\lambda / \Lambda)^{(k-2)}\left[1+C_{2}|\Im \xi|^{2} /(\Re \eta / \Lambda)\right]^{(k-2)}|v| \\
& \quad+(1-\lambda / \Lambda)\left\|\nabla_{\xi} u_{k-1}(\xi, \eta, \cdot, \cdot, \cdot)\right\|_{L^{q}\left(\mathbf{Z}^{d} \times \mathbf{R}, \mathcal{H}\right)},
\end{aligned}
$$

where $C_{q}$ depends only on $q, d$. Hence using Lemma 5.4 , we have by induction from (5.70)-(5.72) that for $k \geq 2$,

$$
\begin{aligned}
& \left\|f_{k}(\xi, \eta, \cdot, \cdot, \cdot)\right\|_{L^{q}\left(\mathbf{Z}^{d} \times \mathbf{R}, \mathcal{H}\right)} \\
& \leq C_{q} k[1+\delta(q)]^{k-2} \Lambda^{-1 / q}\|D \tilde{\mathbf{b}}(\cdot)\|_{\infty} \\
& \quad \times(1-\lambda / \Lambda)^{(k-2)}\left[1+C_{2}|\Im \xi|^{2} /(\Re \eta / \Lambda)\right]^{(k-2)}|v| .
\end{aligned}
$$

The inequality (4.16) follows now from (5.73) and Lemma 5.4 by taking $q$ sufficiently close to 2 so that $1+\delta(q)<(1-\lambda / \Lambda)^{-1 / 2}$.

\section{Appendix A. Diffusion processes with convex potential}

Let $W: \mathbf{R}^{k} \rightarrow \mathbf{R}$ be a $C^{2}$ uniformly convex function such that $W^{\prime \prime}(\cdot)$ satisfies the quadratic form inequality $\lambda I_{k} \leq W^{\prime \prime}(\cdot) \leq \Lambda I_{k}$ for some constants $\lambda, \Lambda>0$. We consider the diffusion process $\phi: \mathbf{R}^{+} \rightarrow \mathbf{R}^{k}$ which is the solution to the SDE initial value problem

$$
d \phi(t)=-\frac{1}{2} \nabla W(\phi(t)) d t+d B(t), \quad t>0, \phi(0)=0,
$$

where $B(\cdot)$ is $k$ dimensional Brownian motion. The invariant measure for the $\operatorname{SDE}($ A.1) is given by

$$
\exp [-W(\phi)] d \phi / \text { normalization, } \quad \phi \in \mathbf{R}^{k} .
$$

We denote the probability space for the stationary process of functions $\phi: \mathbf{R} \rightarrow \mathbf{R}^{k}$ associated with the SDE (A.1) and invariant measure (A.2) by $(\Omega, \mathcal{F}, P)$, and expectation with respect to $(\Omega, \mathcal{F}, P)$ by $\langle\cdot\rangle_{\Omega}$. For $T>0$ let $\phi_{T}:[-T, \infty) \rightarrow \mathbf{R}^{k}$ be defined by $\phi_{T}(t)=\phi(T+t)$, where $\phi(\cdot)$ is the solution to (A.1). The stationary process measure can be obtained by taking the $T \rightarrow \infty$ limit of $\phi_{T}$ as follows:

Lemma A.1. Let $f: \mathbf{R}^{N k} \rightarrow \mathbf{R}$ be a continuous bounded function. Then

$$
\lim _{T \rightarrow \infty}\left\langle f\left(\phi_{T}\left(t_{1}\right), \ldots, \phi_{T}\left(t_{N}\right)\right)\right\rangle=\left\langle f\left(\phi\left(t_{1}\right), \ldots, \phi\left(t_{N}\right)\right)\right\rangle_{\Omega} .
$$


Proof. The diffusion equation corresponding to the SDE (A.1) is given by

$$
\frac{\partial u(\phi, t)}{\partial t}=-\frac{1}{2} \nabla W(\phi) \cdot \nabla u(\phi, t)+\frac{1}{2} \Delta u(\phi, t), \quad t>0 .
$$

The solution to (A.4) with initial data

$$
u(\phi, 0)=u_{0}(\phi), \quad \phi \in \mathbf{R}^{k},
$$

can be written in terms of the Green's function $G: \mathbf{R}^{k} \times \mathbf{R}^{k} \times \mathbf{R}^{+} \rightarrow \mathbf{R}$ as

$$
u(\phi, t)=\int_{\mathbf{R}^{k}} G\left(\phi, \phi^{\prime}, t\right) u_{0}\left(\phi^{\prime}\right) d \phi^{\prime}, \quad \phi \in \mathbf{R}^{k}, t>0 .
$$

Now it is clear that for a continuous bounded function $f: \mathbf{R}^{k} \rightarrow \mathbf{R}$,

$$
\left\langle f\left(\phi_{T}\left(t_{1}\right)\right)\right\rangle=\int_{\mathbf{R}^{k}} G\left(0, \phi^{\prime}, T+t_{1}\right) f\left(\phi^{\prime}\right) d \phi^{\prime} .
$$

Let $\langle\cdot\rangle_{W}$ denote expectation with respect to the invariant measure (A.2) and $L_{W}^{2}\left(\mathbf{R}^{k}\right)$ the corresponding space of square integrable functions $g: \mathbf{R}^{k} \rightarrow \mathbf{R}$ with respect to $\langle\cdot\rangle_{W}$. Letting $[\cdot, \cdot]_{W}$ denote the inner product on $L_{W}^{2}\left(\mathbf{R}^{k}\right)$ we see from (A.7) that for any $\delta$ satisfying $0<\delta<T+t_{1}$,

$$
\begin{aligned}
& \left\langle f\left(\phi_{T}\left(t_{1}\right)\right)\right\rangle=\left[f_{1}, f_{2}\right]_{W}, \\
& \quad \text { where } f_{1}(\phi)=G(0, \phi, \delta) \exp [W(\phi)] \int_{\mathbf{R}^{k}} \exp \left[-W\left(\phi^{\prime}\right)\right] d \phi^{\prime} \\
& \quad \text { and } f_{2}(\phi)=\int_{\mathbf{R}^{k}} G\left(\phi, \phi^{\prime}, T+t_{1}-\delta\right) f\left(\phi^{\prime}\right) d \phi^{\prime} .
\end{aligned}
$$

Since $\left\|f_{2}\right\|_{\infty} \leq\|f\|_{\infty}$ it follows that $f_{2} \in L_{W}^{2}\left(\mathbf{R}^{k}\right)$. We can also easily see that for $\delta>0$ sufficiently small the function $f_{1}$ is in $L_{W}^{2}\left(\mathbf{R}^{k}\right)$. Now we use the fact that the operator $H=-\Delta+\nabla W(\phi) \cdot \nabla$ is self adjoint non-negative definitive on $L_{W}^{2}\left(\mathbf{R}^{k}\right)$ and the constant is an eigenfunction of $H$ with eigenvalue 0 . From the Brascamp-Lieb inequality [4] the operator $H$ acting on the subspace of $L_{W}^{2}\left(\mathbf{R}^{k}\right)$ of functions orthogonal to the constant is bounded below by $\lambda>0$. Furthermore from (A.8), we have that

$$
\left\langle f\left(\phi_{T}\left(t_{1}\right)\right)\right\rangle=\left[f_{1}, e^{-H\left(T+t_{1}-\delta\right) / 2} f\right]_{W},
$$

whence we conclude that

$$
\lim _{T \rightarrow \infty}\left\langle f\left(\phi_{T}\left(t_{1}\right)\right)\right\rangle=\left\langle f_{1}(\phi)\right\rangle_{W}\langle f(\phi)\rangle_{W}=\langle f(\phi)\rangle_{W} .
$$

We have proven (A.3) when $N=1$. The identity (A.3) for $N>1$ can be proven similarly. Assuming $0<t_{1}<t_{2}<\cdots<t_{N}$, we have that

$(\mathrm{A} .11)\left\langle f\left(\phi_{T}\left(t_{1}\right), \ldots, \phi_{T}\left(t_{N}\right)\right)\right\rangle=\left[f_{1}, e^{-H\left(T+t_{1}-\delta\right) / 2} g\right]_{W}$,

$$
\begin{aligned}
\text { where } g\left(\phi_{1}\right)= & \int_{\mathbf{R}^{(N-1) k}} G\left(\phi_{1}, \phi_{2}, t_{2}-t_{1}\right) \cdots G\left(\phi_{N-1}, \phi_{N}, t_{N}-t_{N-1}\right) \\
& \times f\left(\phi_{1}, \ldots, \phi_{N}\right) d \phi_{2} \cdots d \phi_{N} .
\end{aligned}
$$


Letting $T \rightarrow \infty$ in (A.11) we see as before that(A.3) holds.

Next, we wish to obtain a representation of the measure for the probability space $(\Omega, \mathcal{F}, P)$ for the stationary process associated with the SDE (A.1) and invariant measure (A.2). First, we consider the Gaussian case, so there is a symmetric positive definite $k \times k$ matrix $A$ and $k$ dimensional vector $b$ with

$$
W(\phi)=\frac{1}{2} \phi^{*} A \phi-b^{*} \phi, \quad \text { where } \lambda I_{k} \leq A \leq \Lambda I_{k}
$$

The SDE (A.1) is explicitly solvable when $W(\cdot)$ is given by (A.12) with solution

$$
\phi(t)=\int_{0}^{t} e^{-A(t-s) / 2}[b / 2 d s+d B(s)], \quad t \geq 0 .
$$

It is well known that the measure for the stationary process is Gaussian . We can use Lemma A.1 and (A.13) to find formulas for the mean and covariance of $\phi(\cdot)$. Thus, we have that

$$
\begin{aligned}
\langle\phi(t)\rangle_{\Omega} & =A^{-1} b, \\
\operatorname{cov}_{\Omega}\left[\phi\left(t_{1}\right), \phi\left(t_{2}\right)^{*}\right] & =\Gamma\left(t_{2}-t_{1}\right)=A^{-1} e^{-A\left|t_{1}-t_{2}\right| / 2} .
\end{aligned}
$$

The Fourier transform (2.7) of the covariance is therefore given by $\hat{\Gamma}(\theta)=$ $\left[\theta^{2}+A^{2} / 4\right]^{-1}$. Hence, the Gaussian measure corresponding to the covariance is formally given by the expression

$$
\text { (A.15) } \exp \left[-\frac{1}{2} \int_{-\infty}^{\infty}\left|\frac{d \phi(t)}{d t}\right|^{2}+\frac{1}{4}|A \phi(t)-b|^{2} d t\right] \prod_{t \in \mathbf{R}} d \phi(t) / \text { normalization. }
$$

Evidently the measure (A.15) is log concave.

We can obtain a representation of the stationary process measure similar to (A.15) for general $C^{2}$ uniformly convex functions $W(\cdot)$. To see this, we write the solution of (A.4), (A.5) using the Cameron-Martin formula [27] as

$$
\begin{aligned}
u(\phi, t)= & E\left[\operatorname { e x p } \left\{-\frac{1}{2} \int_{0}^{t} \nabla W(B(s)) \cdot d B(s)\right.\right. \\
& \left.\left.-\frac{1}{8} \int_{0}^{t}|\nabla W(B(s))|^{2} d s\right\} u_{0}(B(t)) \mid B(0)=\phi\right]
\end{aligned}
$$

where $B(\cdot)$ is $k$ dimensional Brownian motion. We rewrite (A.16) using Ito's formula

$$
(\mathrm{A} .17) W(B(t))-W(B(0))=\int_{0}^{t} \nabla W(B(s)) \cdot d B(s)+\frac{1}{2} \int_{0}^{t} \Delta W(B(s)) d s .
$$


From (A.16), (A.17), we see that

$$
\begin{aligned}
\text { (A.18) } u(\phi, t)= & e^{W(\phi) / 2} E\left[\exp \left\{-\frac{1}{2} \int_{0}^{t}-\frac{1}{2} \Delta W(B(s))+\frac{1}{4}|\nabla W(B(s))|^{2} d s\right\}\right. \\
& \left.\times e^{-W(B(t)) / 2} u_{0}(B(t)) \mid B(0)=\phi\right] .
\end{aligned}
$$

The identity (A.18) can be alternatively obtained using the Feynman-Kac representation [27] for the solution to the PDE

$$
\frac{\partial v(\phi, t)}{\partial t}=V(\phi) v(\phi, t)+\frac{1}{2} \Delta v(\phi, t), \quad t>0,
$$

with initial data

$$
v(\phi, 0)=v_{0}(\phi), \quad \phi \in \mathbf{R}^{k} .
$$

Thus, we have from the Feynman-Kac formula that

$$
v(\phi, t)=E\left[\exp \left\{\int_{0}^{t} V(B(s)) d s\right\} v_{0}(B(t)) \mid B(0)=\phi\right] .
$$

The formula (A.18) follows now from (A.21) using the fact that if $u(\phi, t)$ is the solution to (A.4), (A.5) then the function $v(\phi, t)=\exp [-W(\phi) / 2] u(\phi, t)$ is the solution to (A.19), (A.20) with

$$
\text { (A.22) } \quad V(\phi)=\frac{1}{4} \Delta W(\phi)-\frac{1}{8}|\nabla W(\phi)|^{2}, \quad v_{0}(\phi)=\exp [-W(\phi) / 2] u_{0}(\phi) .
$$

To obtain the representation for the measure of the stationary process, we use Lemma A.1. Thus from (A.18) the LHS of (A.3) is given by the formula

$$
\begin{aligned}
\left\langle f\left(\phi_{T}\left(t_{1}\right), \ldots, \phi_{T}\left(t_{N}\right)\right)\right\rangle & \\
= & e^{W(0) / 2} E\left[\operatorname { e x p } \left\{-\frac{1}{2} \int_{0}^{T+t_{N}+T^{\prime}}-\frac{1}{2} \Delta W(B(s))\right.\right. \\
& \left.+\frac{1}{4}|\nabla W(B(s))|^{2} d s\right\} e^{-W\left(B\left(T+t_{N}+T^{\prime}\right)\right) / 2} \\
& \left.\times f\left(B\left(T+t_{1}\right), B\left(T+t_{2}\right), \ldots, B\left(T+t_{N}\right)\right) \mid B(0)=0\right],
\end{aligned}
$$

for any $T^{\prime} \geq 0$. Recall now that the Brownian motion measure $B(s), s \geq 0$, has the representation

$$
\text { (A.24) } \exp \left[-\frac{1}{2} \int_{0}^{\infty}\left|\frac{d \phi(s)}{d s}\right|^{2} d s\right] \prod_{s \in \mathbf{R}^{+}} d \phi(s) / \text { normalization } \quad \text { with } \phi(0)=0 .
$$


Hence, on letting $T, T^{\prime} \rightarrow \infty$ in (A.23) and using (A.24), we see that limiting measure defined by $($ A.3 $)$ with expectation $\langle\cdot\rangle_{\Omega}$ has the representation

$$
\exp \left[-\frac{1}{2} \int_{-\infty}^{\infty}\left|\frac{d \phi(t)}{d t}\right|^{2}-\frac{1}{2} \Delta W(\phi(t))+\frac{1}{4}|\nabla W(\phi(t))|^{2} d t\right] \prod_{t \in \mathbf{R}} d \phi(t) .
$$

In the Gaussian case (A.12) the representation (A.25) is equivalent to (A.15) since $\Delta W(\cdot)$ is constant and is therefore part of the normalization constant. The measure (A.25) is $\log$ concave when $W(\cdot)$ is quadratic, but it is easy to see that even if $W(\cdot)$ is a small perturbation of a quadratic the measure is no longer log concave.

\section{Appendix B. The Coulomb dipole gas}

In the lattice Coulomb gas the interaction energy between a particle at $x \in \mathbf{Z}^{d}$ with charge $q_{x}$ and a particle at $y \in \mathbf{Z}^{d}$ with charge $q_{y}$ is $q_{x} q_{y} G(x-y)$ where $G(\cdot)$ is the Green's function for the lattice Laplacian, that is,

$$
-\Delta G(x)=\delta(x), \quad x \in \mathbf{Z}^{d} .
$$

Consider now two dipoles, one along the bond $x \rightarrow x+\mathbf{e}_{i}$ with charge $q_{x}$ at $x$, and the other along the bond $y \rightarrow y+\mathbf{e}_{j}$ with charge $q_{y}$ at $y$. The interaction energy between the two dipoles is given by $q_{x} q_{y} \nabla_{x, i} \nabla_{y, j} G(x-y)$, where $\nabla_{x, i}$ is the difference operator $\nabla_{i}$ of (1.2) applied to the $x$ variable of $G(x-y)$, and similarly $\nabla_{y, j}$ the difference operator $\nabla_{j}$ to the $y$ variable.

In the Gibbs' ensemble for the dipole gas the charge $\rho_{i}(x)$ at $x$ of dipoles on the bond $x \rightarrow x+\mathbf{e}_{i}$ is a compound Poisson variable given by

$$
\rho_{i}(x)=\sum_{r=1}^{\mathcal{N}(x)} Y_{r}(x), \quad Y_{r}(x)= \pm 1 \text { with prob. } \frac{1}{2}, \quad E[\mathcal{N}(x)]=a,
$$

where $\mathcal{N}(x)$ is a Poisson variable and the $Y_{r}(x), r=1,2, \ldots$, are independent Bernoulli. It follows that the characteristic function of $\rho_{k}(x)$ is given by

$$
E\left[\exp \left\{i \theta \rho_{k}(x)\right\}\right]=\exp [a \cos \theta], \quad \theta \in \mathbf{R} .
$$

Let $F: \mathbf{R}^{n} \rightarrow \mathbf{R}$ be a continuous bounded function. The Gibbs' ensemble expectation for $F\left(\rho_{k_{1}}\left(x_{1}\right), \rho_{k_{2}}\left(x_{2}\right) \cdots \rho_{k_{n}}\left(x_{n}\right)\right)$ in the dipole gas is formally given by

$$
\begin{aligned}
& \left\langle F\left(\rho_{k_{1}}\left(x_{1}\right), \rho_{k_{2}}\left(x_{2}\right) \cdots \rho_{k_{n}}\left(x_{n}\right)\right)\right\rangle_{\text {dipole }} \\
& =(\text { normalization })^{-1} E\left[F\left(\rho_{k_{1}}\left(x_{1}\right), \rho_{k_{2}}\left(x_{2}\right) \cdots \rho_{k_{n}}\left(x_{n}\right)\right)\right. \\
& \left.\quad \times \exp \left\{-\frac{1}{2} \sum_{i, j=1}^{d} \sum_{x, y \in \mathbf{Z}^{d}} \rho_{i}(x) \rho_{j}(y) \nabla_{x, i} \nabla_{y, j} G(x-y)\right\}\right],
\end{aligned}
$$


where the variables $\rho_{i}(x), 1 \leq i \leq d, x \in \mathbf{Z}^{d}$ are assumed independent with characteristic function (B.3).

The rigorous study of Coulomb systems proceeds by means of the sineGordon transformation which localizes the interaction. The basic idea is to use the identity

$$
\exp \left[-\frac{1}{2} u A^{-1} u\right]=(\text { constant }) \int_{\mathbf{R}^{n}} \exp \left[i \phi \cdot u-\frac{1}{2} \phi A \phi\right] d \phi,
$$

which holds for any $N \times N$ symmetric positive definite matrix $A$ and $N$ dimensional vector $u$. Observe now that

$$
\begin{aligned}
& \sum_{x, y \in \mathbf{Z}^{d}} \sum_{i, j=1}^{d} \rho_{i}(x) \nabla_{x, i} \nabla_{y, j} G(x-y) \rho_{j}(y) \\
& =\sum_{x, y \in \mathbf{Z}^{d}} \sum_{i, j=1}^{d}\left[\rho_{i}(x)-\rho_{i}\left(x-\mathbf{e}_{i}\right)\right] G(x-y)\left[\rho_{j}(y)-\rho_{j}\left(y-\mathbf{e}_{j}\right)\right] .
\end{aligned}
$$

We conclude that

$$
\sum_{x, y \in \mathbf{Z}^{d}} \sum_{i, j=1}^{d} \rho_{i}(x) \nabla_{x, i} \nabla_{y, j} G(x-y) \rho_{j}(y)=u A^{-1} u,
$$

where

$$
A=-\Delta, \quad u(x)=\sum_{i=1}^{d}\left[\rho_{i}(x)-\rho_{i}\left(x-\mathbf{e}_{i}\right)\right], \quad x \in \mathbf{Z}^{d} .
$$

Let $\psi: \mathbf{Z}^{d} \rightarrow \mathbf{R}^{d}$ be of finite support. Then from (B.5), (B.8) there is a constant $C$ such that

$$
\begin{aligned}
\exp \{ & i \sum_{x \in \mathbf{Z}^{d}} \sum_{j=1}^{d} \psi_{j}(x) \rho_{j}(x) \\
& \left.-\frac{1}{2} \sum_{x, y \in \mathbf{Z}^{d}} \sum_{i, j=1}^{d} \rho_{i}(x) \nabla_{x, i} \nabla_{y, j} G(x-y) \rho_{j}(y)\right\} \\
= & C \int \exp \left\{i \sum_{x \in \mathbf{Z}^{d}} \sum_{j=1}^{d}\left[\nabla_{j} \phi(x)+\psi_{j}(x)\right] \rho_{j}(x)-\frac{1}{2} \sum_{x \in \mathbf{Z}^{d}}|\nabla \phi(x)|^{2}\right\} \\
& \times \prod_{x \in \mathbf{Z}^{d}} d \phi(x) .
\end{aligned}
$$


It follows from (B.3), (B.9) that

$$
\begin{aligned}
& \left\langle\exp \left\{i \sum_{x \in \mathbf{Z}^{d}} \sum_{j=1}^{d} \psi_{j}(x) \rho_{j}(x)\right\}\right\rangle_{\text {dipole }} \\
& =\left\langle\exp \left\{a \sum_{x \in \mathbf{Z}^{d}} \sum_{j=1}^{d} \cos \left[\nabla_{j} \phi(x)+\psi_{j}(x)\right]-\cos \left[\nabla_{j} \phi(x)\right]\right\}\right\rangle
\end{aligned}
$$

where the measure on the RHS of (B.10) is the massless field measure (1.1) with $V(\cdot)$ given by (1.5). We conclude from (B.10) that the first and second moment dipole gas expectations are related to massless field expectations by

$$
\begin{aligned}
\left\langle\rho_{j}(x)\right\rangle_{\text {dipole }} & =0, \\
\left\langle\rho_{j}(x) \rho_{k}(y)\right\rangle_{\text {dipole }} & =-a^{2}\left\langle\sin \left\{\nabla_{j} \phi(x)\right\} \sin \left\{\nabla_{k} \phi(y)\right\}\right\rangle, \quad x \neq y .
\end{aligned}
$$

Expectations for the Gibbs' ensemble for just two dipoles, one on the bond $x \rightarrow x+\mathbf{e}_{i}$ and the other on the bond $y \rightarrow y+\mathbf{e}_{j}$ is given similarly to (B.4) by

$$
\begin{aligned}
& \left\langle F\left(\rho_{i}(x), \rho_{j}(y)\right)\right\rangle_{x, y, \text { dipole }} \\
& =(\text { normalization })^{-1} E\left[F\left(\rho_{i}(x), \rho_{j}(y)\right)\right. \\
& \left.\quad \times \exp \left\{-\alpha \rho_{i}(x)^{2} / 2-\alpha \rho_{j}(y)^{2} / 2-\rho_{i}(x) \rho_{j}(y) \nabla_{x, i} \nabla_{y, j} G(x-y)\right\}\right],
\end{aligned}
$$

where $\alpha=\left.\nabla_{x, i} \nabla_{y, i} G(x-y)\right|_{x=y}=2 G(0)-G\left(\mathbf{e}_{i}\right)-G\left(-\mathbf{e}_{i}\right)=1 / d$ from (B. 1$)$. The variables $\rho_{i}(x), \rho_{j}(y)$ are independent and have characteristic function given by (B.3). Thus the probability measure (B.12) is obtained from the measure (B.4) by retaining only the compound Poisson variables $\rho_{i}(x), \rho_{j}(y)$ and setting all other variables to zero. We can estimate second moments for the measure (B.12) when $|x-y| \gg 1$ by using the fact that $\nabla_{x, i} \nabla_{y, j} G(x-y) \simeq$ $1 /|x-y|^{d}$. Thus,

$$
\begin{aligned}
&\left\langle\rho_{i}(x)\right.\left.\rho_{j}(y)\right\rangle_{x, y, \text { dipole }} \\
&=-\nabla_{x, i} \nabla_{y, j} G(x-y) \\
& \quad \times\left\{E\left[\rho_{i}(x)^{2} \exp \left\{-\alpha \rho_{i}(x)^{2} / 2\right\}\right] / E\left[\exp \left\{-\alpha \rho_{i}(x)^{2} / 2\right\}\right]\right\}^{2} \\
& \quad+O\left(1 /|x-y|^{2 d}\right) .
\end{aligned}
$$

We have now from (B.3), (B.5) that

$$
E\left[\exp \left\{-\alpha \rho_{i}(x)^{2} / 2\right\}\right]=E\left[e^{a \cos (\sqrt{\alpha} Z)}\right]=1+a e^{-\alpha / 2}+O\left(a^{2}\right),
$$

where $Z$ is the standard normal variable. It follows from (B.13), (B.14) that

$$
\left\langle\rho_{i}(x) \rho_{j}(y)\right\rangle_{x, y, \text { dipole }}=-\nabla_{x, i} \nabla_{y, j} G(x-y) a^{2} e^{-1 / d}[1+O(a)] .
$$

We compare the second moment (B.15) for the two dipole system to the corresponding second moment for the complete system with measure (B.4). 
To do this we use the identity (B.11) and make the quadratic approximation $V(z) \simeq(1+a)|z|^{2} / 2-d a, z \in \mathbf{R}^{d}$ for the function (1.5). In that case the massless field expectation (1.1) for (1.5) is approximately the Gaussian expectation

$$
\left\langle\sin \left\{\nabla_{i} \phi(x)\right\} \sin \left\{\nabla_{j} \phi(y)\right\}\right\rangle \simeq\left\langle\sin \left\{\nabla_{i} \phi(x)\right\} \sin \left\{\nabla_{j} \phi(y)\right\}\right\rangle_{\text {Gaussian }} .
$$

The RHS of (B.16) can be explicitly computed by using the identity

$$
\begin{aligned}
& \left\langle\exp \left[i \sum_{z \in \mathbf{Z}^{d}} f(z) \phi(z)\right]\right\rangle_{\text {Gaussian }} \\
& =\exp \left[-\frac{1}{2(1+a)} \sum_{z, z^{\prime} \in \mathbf{Z}^{d}} f(z) G\left(z-z^{\prime}\right) f\left(z^{\prime}\right)\right] .
\end{aligned}
$$

We see from (B.17) that

$$
\begin{aligned}
& \left\langle\sin \left\{\nabla_{i} \phi(x)\right\} \sin \left\{\nabla_{j} \phi(y)\right\}\right\rangle_{\text {Gaussian }} \\
& \quad=\exp \left[-\frac{1}{(1+a) d}\right] \frac{1}{1+a} \nabla_{x, i} \nabla_{y, j} G(x-y)+O\left(\frac{1}{|x-y|^{2 d}}\right) .
\end{aligned}
$$

Observing now that if $d \geq 1$ then

$$
\exp \left[\frac{a}{(1+a) d}\right] \frac{1}{1+a}<1 \text { for } a>0,
$$

we conclude from (B.11), (B.15), (B.18) that if $a$ is sufficiently small, then $\left|\left\langle\rho_{i}(x) \rho_{j}(y)\right\rangle_{\text {dipole }}\right|<\left|\left\langle\rho_{i}(x) \rho_{j}(y)\right\rangle_{x, y \text {,dipole }}\right|$ for $|x-y| \gg 1$. Hence, we have verified the effect of screening within the Gaussian approximation for the function $V(\cdot)$.

Finally, we justify the Gaussian approximation for $V(\cdot)$ in the previous paragraph by obtaining the expansion of $\mathbf{a}_{\text {hom }}$ in the Naddaf-Spencer homogenized PDE (1.6) with $V(\cdot)$ given by (1.5) to first order in $a$. To do this, we recall that $\mathbf{a}_{\text {hom }}=q(0,0)$ where $q(\xi, \eta)$ is given by (4.3) and $\mathbf{a}(\cdot)$ is diagonal with $\mathbf{a}_{i, i}(\phi)=1+a \cos \left(\nabla_{i} \phi(0,0)\right), i=1, \ldots, d$. It is easy to see that the second term $\left\langle\mathbf{a}(\cdot) \partial_{\xi} \Phi(\xi, \eta)\right\rangle$ on the RHS of (4.3) is $O\left(a^{2}\right)$, whence we conclude that

$$
\mathbf{a}_{\mathrm{hom}}=\left(1+a^{\prime}\right) I_{d}+O\left(a^{2}\right), \quad \text { where } a^{\prime}=a\left\langle\cos \left(\nabla_{1} \phi(0)\right)\right\rangle_{a},
$$

and $\langle\cdot\rangle_{a}$ denotes expectation with respect to the massless measure (1.1) with $V(\cdot)$ given by (1.5). It is easy to see, as in the proof of Theorem 3.1 of [19], that $\left\langle\cos \left(\nabla_{1} \phi(0)\right)\right\rangle_{a}>0$, whence $a^{\prime}>0$. We shall show using the method employed in [10] to construct the measures (1.1) that

$$
\left\langle\cos \left(\nabla_{1} \phi(0)\right)\right\rangle_{a}=\left\langle\cos \left(\nabla_{1} \phi(0)\right)\right\rangle_{0}+O(a) .
$$

We have that the Gaussian expectation $\left\langle\cos \left(\nabla_{1} \phi(0)\right)\right\rangle_{0}=\exp [-\{2 G(0)-$ $\left.\left.G\left(\mathbf{e}_{1}\right)-G\left(-\mathbf{e}_{1}\right)\right\} / 2\right]=\exp [-1 / 2 d]$. Hence, from (B.20) it follows that $\mathbf{a}_{\text {hom }}=$ $\left(1+a e^{-1 / 2 d}\right) I_{d}+O\left(a^{2}\right)$, which is different from what we obtained in the naive 
argument of the previous paragraph that yielded $\mathbf{a}_{\mathrm{hom}}=1+a$ to first order in $a$. Note however that the screening argument there continues to work since $\mathbf{a}_{\mathrm{hom}}>I_{d}$.

To prove (B.21), we obtain an inequality for the measure (5.37), which is uniform as $Q \rightarrow \mathbf{Z}^{d}$ and $m \rightarrow 0$. Letting $\langle\cdot\rangle_{a, \Omega_{Q, m}}$ denote expectation with respect to the measure $(5.37)$ with $V(\cdot)$ given by $(1.5)$, we have from the fundamental theorem of calculus that

$$
\begin{aligned}
& \left\langle\cos \left(\nabla_{1} \phi(0)\right)\right\rangle_{a, \Omega_{Q, m}} \\
& =\left\langle\cos \left(\nabla_{1} \phi(0)\right)\right\rangle_{0, \Omega_{Q, m}} \\
& \quad+a \int_{0}^{1} d t \sum_{j=1}^{d} \sum_{x \in Q} \operatorname{cov}_{t a, \Omega_{Q, m}}\left[\cos \left(\nabla_{1} \phi(0)\right), \cos \left(\nabla_{j} \phi(x)\right)\right] .
\end{aligned}
$$

By translation invariance of the measure (5.37), we have that

$$
\begin{aligned}
& \sum_{x \in Q} \operatorname{cov}_{t a, \Omega_{Q, m}}\left[\cos \left(\nabla_{1} \phi(0)\right), \cos \left(\nabla_{j} \phi(x)\right)\right] \\
& =\frac{1}{L^{d}} \operatorname{cov}_{t a, \Omega_{Q, m}}\left[F_{1}(\phi), F_{j}(\phi)\right],
\end{aligned}
$$

where the function $F_{j}, j=1, \ldots, d$ of periodic fields $\phi: Q \rightarrow \mathbf{R}$ is given by

$$
F_{j}(\phi)=\sum_{x \in Q} \cos \left(\nabla_{j} \phi(x)\right) .
$$

We can estimate the RHS of (B.23) by using (1.3). Thus, we have that

$$
\operatorname{Var}_{t a, \Omega_{Q, m}}\left[F_{j}(\phi)\right] \leq\left\langle\left[d F_{j}(\phi),\left\{-(1-a) \Delta+m^{2}\right\}^{-1} d F_{j}(\phi)\right]\right\rangle_{t a, \Omega_{Q, m}}
$$

where $\Delta$ is the Laplacian for the periodic cube $Q$. Observe now that $d F_{j}(x ; \phi)=\nabla_{j}^{*} G_{j}(x, \phi), x \in Q$, where $G_{j}(x, \phi)=\sin \left(\nabla_{j} \phi(x)\right), x \in Q$. It follows that

$$
\begin{aligned}
& {\left[d F_{j}(\phi),\left\{-(1-a) \Delta+m^{2}\right\}^{-1} d F_{j}(\phi)\right]} \\
& \quad=\left[G_{j}(\phi), \nabla_{j}\left\{-(1-a) \Delta+m^{2}\right\}^{-1} \nabla_{j}^{*} G_{j}(\phi)\right] \leq\left\|G_{j}(\phi)\right\|_{2}^{2} \leq L^{d} .
\end{aligned}
$$

We conclude from (B.22)-(B.26) and Theorem 3.1 of [19] that

$$
0 \leq\left\langle\cos \left(\nabla_{1} \phi(0)\right)\right\rangle_{a, \Omega_{Q, m}}-\left\langle\cos \left(\nabla_{1} \phi(0)\right)\right\rangle_{0, \Omega_{Q, m}} \leq a d
$$

Acknowledgments. The authors would like to thank Tom Hurd and Tom Spencer for helpful conversations. The research of Arash Fahim was supported by NSF grant DMS-1209519. 


\section{REFERENCES}

[1] D. Aronson, Bounds for the fundamental solution of a parabolic equation, Bull. Amer. Math. Soc. (N.S.) 73 (1967), 890-896. MR 0217444

[2] G. Bal, J. Garnier, S. Motsch and V. Perrier, Random integrals and correctors in homogenization, Asymptot. Anal. 59 (2008), 1-26. MR 2435670

[3] C. Boldrighini, R. Minlos and A. Pellegrinotti, Random walks in quenched i.i.d. space-time environments are always a.s. diffusive, Probab. Theory Related Fields 129 (2004), 133-156. MR 2052866

[4] H. Brascamp and E. Lieb, On extensions of the Brunn-Minkowski and PrékopaLeindler theorems, including inequalities for log concave functions, and with an application to the diffusion equation, J. Funct. Anal. 22 (1976), 366-389. MR 0450480

[5] D. Brydges, Lectures on the renormalisation group, Statistical mechanics, IAS/Park City Math. Ser., vol. 16, Amer. Math. Soc., Providence, 2009, pp. 7-93. MR 2523458

[6] D. Brydges and H.-T. Yau, Grad $\phi$ perturbations of massless Gaussian fields, Comm. Math. Phys. 129 (1990), 351-392. MR 1048698

[7] L. Caffarelli and P. Souganidis, Rates of convergence for the homogenization of fully nonlinear uniformly elliptic pde in random media, Invent. Math. 180 (2010), 301-360. MR 2609244

[8] R. Carmona and M. Tehranchi, Interest rate models: An infinite dimensional stochastic analysis perspective, Springer-Verlag, Berlin-Heidelberg, 2006. MR 2235463

[9] G. Checkin, A. Piatnitski and A. Shamaev, Homogenization: Methods and applications, Translations of Mathematical Monographs, vol. 234, Amer. Math. Soc., Providence, 2007. MR 2337848

[10] J. Conlon, PDE with random coefficients and Euclidean field theories, J. Stat. Phys. 114 (2004), 933-958. MR 2082201

[11] J. Conlon, Greens functions for elliptic and parabolic equations with random coeffcients II, Trans. Amer. Math. Soc. 356 (2004), 4085-4142. MR 2058840

[12] J. Conlon and A. Fahim, Strong convergence to the homogenized limit of parabolic equations with random coefficients, Trans. Amer. Math. Soc. 367 (2015), 3041-3093. MR 3314801

[13] J. Conlon and A. Fahim, Strong convergence to the homogenized limit of elliptic equations with random coefficients II, Bull. Lond. Math. Soc. 45 (2013), 973-986. MR 3104989

[14] J. Conlon and T. Spencer, Strong convergence to the homogenized limit of elliptic equations with random coefficients, Trans. Amer. Math. Soc. 366 (2014), 1257-1288. MR 3145731

[15] T. Delmotte and J. Deuschel, On estimating the derivatives of symmetric diffusions in stationary random environment, with applications to $\nabla \phi$ interface model, Probab. Theory Related Fields 133 (2005), 358-390. MR 2198017

[16] J. Dimock, Infinite volume limit for the dipole gas, J. Stat. Phys. 135 (2009), 393-427. MR 2531084

[17] J. Dimock and T. Hurd, A renormalization group analysis of correlation functions for the dipole gas, J. Stat. Phys. 66 (1992), 1277-1318. MR 1156405

[18] D. Dolgopyat, G. Keller and C. Liverani, Random walk in Markovian environment, Ann. Probab. 36 (2008), 1676-1710. MR 2440920

[19] J. Fröhlich and Y. M. Park, Correlation inequalities and the thermodynamic limit for classical and quantum continuous systems, Comm. Math. Phys. 59 (1978), 235-266. MR 0496191

[20] T. Funaki and H. Spohn, Motion by mean curvature from the Ginzburg-Landau $\nabla \phi$ interface model, Comm. Math. Phys. 185 (1997), 1-36. MR 1463032 
[21] K. Gawedzki and A. Kupiainen, Lattice dipole gas and $(\nabla \phi)^{4}$ models at long distances: Decay of correlations and scaling limit, Comm. Math. Phys. 92 (1984), 531553. MR 0736410

[22] G. Giacomin, S. Olla and H. Spohn, Equilibrium fluctuations for $\nabla \phi$ interface model, Ann. Probab. 29 (2001), 1138-1172. MR 1872740

[23] A. Gloria and F. Otto, An optimal variance estimate in stochastic homogenization of discrete elliptic equations, Ann. Probab. 39 (2011), 779-856. MR 2789576

[24] A. Gloria and F. Otto, An optimal error estimate in stochastic homogenization of discrete elliptic equations, Ann. Appl. Probab. 22 (2012), 1-28. MR 2932541

[25] A. Gloria, S. Neukamm and F. Otto, Quantification of ergodicity in stochastic homogenization: Optimal bounds via spectral gap on glauber dynamics, Invent. Math. 199 (2015), 455-515. MR 3302119

[26] B. F. Jones, A class of singular integrals, Amer. J. Math. 86 (1964), 441-462. MR 0161099

[27] I. Karatzas and S. Shreve, Brownian motion and stochastic calculus, 2nd ed., Graduate Texts in Mathematics, vol. 113, Springer-Verlag, New York, 1991. MR 1121940

[28] S. Kozlov, Averaging of random structures, Dokl. Akad. Nauk SSSR 241 (1978), 10161019. MR 0510894

[29] S. Kozlov, The method of averaging and walks in inhomogeneous environment, Russian Math. Surveys 40 (1985), 73-145. MR 0786087

[30] C. Landim, S. Olla and H. T. Yau, Convection-diffusion equation with space-time ergodic random flow, Probab. Theory Related Fields 112 (1998), 203-220. MR 1653837

[31] D. Marahrens and F. Otto, Annealed estimates on the Green function, Probab. Theory Related Fields 163 (2015), 527-573. MR 3418749

[32] N. Meyers, An $L^{p}$ estimate for the gradient of solutions of second order elliptic divergence equations, Ann. Sc. Norm. Super. Pisa Cl. Sci. (5) 17 (1963), 189-206. MR 0159110

[33] J. Moser, On Harnack's inequality for parabolic differential equations, Comm. Pure Appl. Math. 14 (1961), 577-591.

[34] J. C. Mourrat, Kantorivich distance in the martingale CLT and quantitative homogenization of parabolic equations with random coefficients, Probab. Theory Related Fields 160 (2014), 279-314. MR 3256815

[35] D. Mugler, Green's functions for the finite difference heat, Laplace and wave equations, Anniversary volume on approximation theory and functional analysis (Oberwolfach, 1983), Internat. Schriftenreihe Numer. Math., vol. 65, Birkhäuser, Basel, 1984, pp. 543554. MR 0820550

[36] A. Naddaf and T. Spencer, On homogenization and scaling limit of some gradient perturbations of a massless free field, Comm. Math. Phys. 183 (1997), 55-84. MR 1461951

[37] A. Naddaf and T. Spencer, Estimates on the variance of some homogenization problems, preprint, 1998.

[38] D. Nualart, The Malliavin calculus and related topics, 2nd ed., Springer Verlag, Berlin, 2005. MR 2200233

[39] G. Papanicolaou and S. Varadhan, Boundary value problems with rapidly oscillating random coefficients, Random fields, vol. 2, Coll. Math. Soc. Janos Bolya, vol. 27, North Holland, Amsterdam, 1981, pp. 835-873. MR 0712714

[40] R. Rhodes, On homogenization of space-time dependent and degenerate random flows, Stochastic Process. Appl. 117 (2007), 1561-1585. MR 2353040

[41] E. Stein, Singular integrals and differentiability properties of functions, Princeton University Press, Princeton, NJ, 1970. MR 0290095

[42] V. Yurinskii, Averaging of symmetric diffusion in random medium, Sibirsk. Mat. Zh. 27 (1986), 167-180. MR 0867870 
[43] V. Zhikov, S. Kozlov and O. Oleinik, Homogenization of differential operators and integral functionals, Springer Verlag, Berlin, 1994. MR 1329546

Joseph G. Conlon, Department of Mathematics, University of Michigan, Ann ARBor, MI 48109-1109, USA

E-mail address: conlon@umich.edu

Arash Fahim, Department of Mathematics, Florida State University, TallaHASSEE, FL 32306, USA

E-mail address: fahim@math.fsu.edu 